\title{
THE CONTRIBUTION OF GALAXY CLUSTERS TO THE SOFT X-RAY BACKGROUND
}

\author{
A. BLANCHARD \\ Observatoire de Paris-Section de Meudon, Place Janssen, F-92195, Meudon, France; and Université de Paris VII \\ K. WACHTER \\ Department of Statistics, University of California, Berkeley, CA 94720 \\ A. E. EVRARD \\ Department of Physics, University of Michigan, Ann Arbor, MI 48109 \\ AND \\ JOSEPH SILK \\ Departments of Astronomy and Physics, and Center of Particle Astrophysics, University of California, Berkeley, CA 94720 \\ Received 1991 May 13; accepted 1991 November 20
}

\begin{abstract}
We examine models for the expected contribution to the X-ray background (XRB) from clusters of galaxies in a flat universe with a spectrum of fluctuations corresponding to the standard cold dark matter picture and for a power-law spectrum $P(k) \propto k^{n}$ with $n=-2$. We consider a self-similar scaling model for clusters proposed by Kaiser, along with several variants. The self-similar model produces an appreciable fraction of the soft X-ray background. However, the predicted luminosity function overproduces low-luminosity clusters and expected number counts exceed observations. Incompleteness due to spatial extension of the sources is unlikely to account for these discrepancies. Photon statistics from synthesized maps of deep Einstein fields show a granularity similar to that deduced by Hamilton and Helfand from the Einstein data but overproduces by a large factor the number of discrete sources observed as clusters, and the fluctuation limit derived from the recent correlation analyses of the background. This occurs even though a large fraction of cluster sources may be missed by detection algorithms geared toward detection of point sources.

Two alternative models are designed to be consistent with the local luminosity function data. For these, we generate expected deep images for the ROSAT satellite. We predict that a substantial fraction of extragalactic component of the soft (0.1-2.4 keV) XRB might be due to clusters. Clusters of galaxies should be a significant source of background at energies around $2 \mathrm{keV}$ and are likely to contribute up to $30 \%$ of the unresolved component. They should produce fluctuations at a level close to present limits. These sources are extended and might easily be missed by use of a point source algorithm for detection. Furthermore, we find that the clusters' number counts as well as their contribution in the soft ROSAT energy ranges are higher in the $n=-2$ model than in CDM models. ROSAT observations might therefore help in differentiating between these models.

Subject headings: dark matter - diffuse radiation - galaxies: clustering - X-rays: galaxies
\end{abstract}

\section{INTRODUCTION}

The X-ray background, discovered by Giacconi et al. (1962), was the first cosmic background detected, predating by several years the discovery of the microwave background (Penzias \& Wilson 1965). Its origin has been discussed at length in the literature (see Boldt 1987 for a review), with a particular focus on the question of the relative contributions from active galaxies, high-redshift quasars and hot gas in galaxy clusters. Its origin still remains unknown. At energies well above $3 \mathrm{keV}$, it is possible that the background originates from active galaxies (Schwartz \& Tucker 1988; Morisawa \& Takahara 1989; Setti \& Woltjer 1991), despite the so-called spectral paradox (Boldt 1987). Hot uniform IGM models (Cowsik \& Kobetich 1972) have been definitively ruled out by the limit on the Compton parameter $y$ obtained by COBE (Mather et al. 1990). It is also possible that most of the background originates from a highredshift population (Fabian et al. 1990; Collin-Souffrin 1991). The contribution above $3 \mathrm{keV}$ from rich clusters has been estimated to be less than 10\% (McKee et al. 1980; Piccinotti et al. 1982). Indeed, the extragalactic background in the 3-100 $\mathrm{keV}$ domain has a component characterized by a thermal bremsstrahlung spectrum of $40 \mathrm{keV}$ (Marshall et al. 1980), much hotter than the typical temperatures of rich clusters $\sim 7$
$\mathrm{keV}$, so that clusters cannot provide most of the high-energy background. However, it has been pointed out that lowluminosity and low-temperature clusters could contribute more than the above estimate in the energy range $2-10 \mathrm{keV}$, (Stottlemeyer \& Boldt 1984).

At energies below $3 \mathrm{keV}$, the origin and intensity of the extragalactic X-ray background is less certain (McCammon \& Sanders 1990). In the Einstein band 1-3 keV the intensity of the total flux extrapolated from a $40 \mathrm{keV}$ bremsstrahlung fit to the diffuse $3-100 \mathrm{keV}$ background is around $6 \times 10^{-12} \mathrm{erg} \mathrm{s}^{-1}$ $\mathrm{cm}^{-2} \mathrm{deg}^{-2}$. However, direct estimation from analysis of Einstein fields leads to a value higher by $\sim 50 \%$ (Wu et al. 1991). This is confirmed by the shape of the spectrum measured by ROSAT (Hasinger, Schmidt, \& Trümper 1991).

Another important constraint on the possible origin of the $\mathrm{X}$-ray background comes from its homogeneity. From a fluctuation analysis of deep Einstein fields, Hamilton \& Helfand (1987) have shown that it is unlikely that the $1-3 \mathrm{keV}$ background originates from known classes of point sources by extrapolating the $\log N(>S)$ graph down to lower fluxes. The deepest counts from ROSAT show that $45 \%$ of the background at $1 \mathrm{keV}$ is resolved into pointlike sources (Hasinger 1992). While a high-resolution ROSAT image (Shanks et al. 
1991) reveals that quasars contribute at least $30 \%$ of the X-ray background at $1 \mathrm{keV}$, the Hamilton-Helfand analysis strongly suggests that normal QSOs cannot produce the whole intensity and that an unknown population has to be introduced in order to explain the residual fraction of the soft X-ray background. If the background originates from such an additional population of point sources, then these sources should be numerous (at least several thousand $\mathrm{deg}^{-2}$ ). However, such sources do not correspond to any known population of X-ray sources. Barcons \& Fabian (1989) extended the fluctuation analysis and claim that the background has a small, positive correlation function on scales of a few arcminutes. A reanalysis by Soltan (1991) shows that detector noise dominated the Einstein IPC correlations, and ROSAT data shows that the X-ray background autocorrelation function has a smaller value (Hasinger et al. 1991). The lack of significant clustering of photons above $\sim 5^{\prime}$ may serve as a useful constraint on the origin of the diffuse XRB in the Einstein 1-3 keV band, as only sources at high redshift could produce this small a degree of clustering.

The contribution of clusters is small in the high-energy band and cannot explain the total background at energies above a few $\mathrm{keV}$. However, this contribution is likely to be higher in the soft energy band than in the better-studied higher bands, as Silk \& Tarter (1973) have contended. Schaeffer \& Silk (1988) also point out that a major component of the soft X-ray background may be the population of clusters and groups of galaxies, with cooler temperature, low-mass systems contributing preferentially at softer X-ray energies. The aim of this paper is to examine in detail such a possibility.

\section{A MODEL FOR THE CLUSTER CONTRIBUTION TO THE XRB}

$\mathrm{X}$-ray emission from clusters is well-described by thermal bremsstrahlung from an optically thin source with a single temperature $T_{X}$. Assuming that the total X-ray luminosity $L_{X}$ and the temperature $T_{X}$ depend only on the mass $M$ and redshift epoch $z$, the background contribution from clusters in a given energy band $E_{1}-E_{2}$ can be written as

$$
\phi_{E_{1}-E_{2}}=\int_{0}^{+\infty} d V(z) \int_{0}^{+\infty} d M \Phi(M, z) \frac{L_{E_{1}-E_{2}}(M, z)}{4 \pi D_{\text {lum }}^{2}(z)}
$$

with

$$
\begin{aligned}
L_{E_{1}-E_{2}}(M, z)=\left[\tilde{g}\left(E_{1} / k T_{X}\right) e^{-E_{1} / k T_{X}(1+z)}\right. & \\
& \left.-\tilde{g}\left(E_{2} / k T_{X}\right) e^{-E_{2} / k T_{X}(1+z)}\right] L_{X}(M, z) .
\end{aligned}
$$

Here $\Phi$ is the mass function of clusters at redshift $z, D_{\text {lum }}$ is the luminosity distance, $V$ stands for the volume element at redshift $z$, and $\tilde{g}(x)$ is the velocity-averaged Gaunt factor. For simplicity, the Gaunt factor will be taken to be constant, which is a fair approximation over the $0.1-3 \mathrm{keV}$ energy range within which we are interested.

\subsection{Cluster Properties from a Spherical Collapse Model}

In order to evaluate the X-ray flux from galaxy clusters, we need a model for gas in clusters and a mass function for the clusters at any redshift. The spherical, "top-hat" model (Gunn \& Gott 1972; Peebles 1980) allows us to establish the virial temperature $T_{V}$ as a function of the mass of the cluster and its redshift. The gas distribution will be assumed to be isothermal with a temperature given by the virial temperature. A fluctuation, for which the density contrast extrapolated linearly up to now is $\delta$, reaches its maximum expansion, with a radius $R_{m}$ at an epoch $t_{m}$ corresponding to redshift $z_{m}$, when

$$
1+z_{m}=\frac{\delta}{1.06} \text {. }
$$

Virial equilibrium is approximately achieved after one dynamical time at the epoch $t_{V}=2 t_{m}$, corresponding to the redshift $z$; therefore the linear contrast density $\delta_{V}$ necessary to reach this regime

$$
\delta_{V}=1.68(1+z) .
$$

The fluctuation has then collapsed by some factor $f_{c}$ and the contrast density reaches the value

$$
1+\Delta_{V}=\frac{9 \pi^{2}}{4 f_{c}^{3}} \approx 22.2 f_{c}^{-3}
$$

The final radius is

$$
R_{V}=f_{c} R_{m}=1.68 f_{c} M_{15}^{1 / 3}(1+z)^{-1} h^{-2 / 3} \mathrm{Mpc} .
$$

Assuming an isothermal sphere, the one-dimensional velocity dispersion $\sigma$ in terms of the gravitational constant $G$ is

$$
\sigma=\sqrt{\frac{1}{2} \frac{G M}{R_{V}}}=1128\left(\frac{0.5}{f_{c}}\right)^{1 / 2} M_{15}^{1 / 3} h^{1 / 3}(1+z)^{1 / 2} \mathrm{~km} \mathrm{~s}^{-1} .
$$

The corresponding temperature of the gas is

$$
T_{V}=\frac{\mu m_{p}}{k} \sigma^{2}=3.91 f_{c}^{-1} M_{15}^{2 / 3}(1+z) h^{2 / 3} \mathrm{keV} .
$$

For a collapse factor $f_{c}$ of 0.62 , this relation is in excellent agreement with the results from numerical simulations (Evrard 1990). This value is used hereafter.

The bolometric luminosity from bremsstrahlung emission of the cluster depends on its density profile

$$
L_{X}=\int_{0}^{+\infty} n^{2}(R) T^{1 / 2} 4 \pi R^{2} d R
$$

The density profile for the X-ray gas in clusters is modeled by the isothermal $\beta$-form

$$
n(R)=\frac{n_{c}}{\left[1+\left(R / R_{c}\right)^{2}\right]^{3 \beta / 2}} \approx(1-\beta) \Delta_{V} \rho_{b} f_{M}\left(\frac{R}{R_{V}}\right)^{3 \beta},
$$

where $f_{M}$ is the fraction of the cosmological gas going into the intracluster medium (ICM hereafter). In this model, the total luminosity at a fixed mass is dominated by the short-scale cutoff introduced by the core radius $R_{c}$

$$
L_{X} \propto \int_{0}^{+\infty} n^{2}(R) T^{1 / 2} R^{2} d R \propto f_{M}^{2} M^{2 \beta} T^{1 / 2} R_{c}^{3(1-2 \beta)}
$$

when $\beta$ is larger than 0.5 . This latter constraint is always true for the density profiles of observed clusters (Jones \& Forman 1984).

\subsection{Cluster Evolution}

The top-hat model allows us to derive the evolution of properties with redshift as discussed by Kaiser (1986)

$$
\begin{gathered}
R_{V} \propto(1+z)^{-1} M^{1 / 3}, \\
T_{V} \propto(1+z) M^{2 / 3}, \\
L_{X} \propto(1+z)^{3.5} M^{4 / 3}\left(\frac{R_{c}}{R_{V}}\right)^{3(1-2 \beta)} .
\end{gathered}
$$


The evolution and scaling of the core radius is highly uncertain, and this uncertainty limits our knowledge of the total X-ray luminosity. It is important to keep in mind that the luminosity is governed by the core properties. The simplest model proposed by Kaiser (1986) is to suppose that the mass distribution is self-similar:

$$
\frac{R_{c}}{R_{V}}=\text { const }, \quad f_{m}=\text { const . }
$$

Then the evolution of the X-ray luminosity is independent of $\beta$ :

$$
L_{X} \propto(1+z)^{3.5} M^{4 / 3} .
$$

The formation of a core in the gas density distribution is determined by several, poorly understood, competing processes. For example, the centers of some rich clusters have cooling times comparable to or less than the Hubble time. The core radius of the $\mathrm{X}$-ray gas might then be determined by the radius at which the cooling time just exceeds the Hubble time. This would modify the above relation to $R_{c} \propto M^{1 / 6}$. However, substantial cooling may occur in substructures before the gas attains its final profile. This could easily modify the relation between mass and core radius. Also, preheating of the central gas could keep it from collapsing to high densities, effectively preventing cooling and generating a larger core radius. Given the large degree of uncertainty, we choose to consider two simple cases-self-similar scaling $R_{c} \propto M^{1 / 3}$ and a constant core $R_{c}=$ const.

To allow models more general than the self-similar case given by equation (11), we parameterize the luminosity-mass relation as a power law

$$
L_{X}=L_{15}(1+z)^{s} M^{p}
$$

and different values of $p$ and $s$ will be considered. Values of $p$ steeper than the self-similar value $4 / 3$ may represent, for example, models in which the cluster ICM fraction is an increasing function of cluster richness. There is some observational evidence supporting this possibility (David et al. 1990). The normalization of the above relation is obtained by matching to the local abundance of X-ray clusters (see Table 1 below). The cluster gas density profiles are assumed to follow the form in equation (6) with

$$
\beta=\frac{2}{3},
$$

the median value for the clusters in the sample of Jones \& Forman (1984).

\subsection{Cluster Mass Function}

In order to derive the contribution of clusters to the background, we need to know the mass function at any redshift. The formula of Press \& Schechter (1974) (hereafter PS) gives a simple prescription of the mass function corresponding to a given power spectrum. In addition, the PS mass function is in

TABLE 1

MODEL PARAMETERS

\begin{tabular}{lccccccc}
\hline \hline Model & $n$ & $L_{15}{ }^{\mathrm{a}}$ & $p^{\mathrm{a}}$ & $s^{\mathrm{a}}$ & $r_{0}{ }^{\mathrm{b}}$ & $u^{\mathrm{b}}$ & $v^{\mathrm{b}}$ \\
\hline CDMSS $\ldots \ldots \ldots \ldots \ldots \ldots$ & -1 & 5.5 & $4 / 3$ & $7 / 2$ & 300 & $1 / 3$ & -1 \\
CDMP3 $\ldots \ldots \ldots \ldots \ldots \ldots$ & -1 & 5.5 & 3 & $7 / 2$ & 300 & 0 & 0 \\
XCDM $\ldots \ldots \ldots \ldots \ldots \ldots$ & -2 & 7 & $11 / 6$ & $11 / 4$ & 300 & $-1 / 6$ & $-1 / 4$ \\
\hline
\end{tabular}

${ }^{\text {a }}$ Bolometric luminosity in $10^{44} \mathrm{ergs} \mathrm{s}^{-1}$ scaling as $L_{\mathrm{bol}}=L_{15} M^{p}(1+z)^{s}$.

b Core radius in kpc scaling as $r_{c}=r_{0} M^{u}(1+z)^{v}$. good agreement with numerical simulations (Efstathiou et al. 1988; Bond et al. 1991). As it is well known, the integral of the simplest form of the PS mass function provides only half of the mean density. The mass function of nonlinear objects must account for all the mass in the universe and therefore the PS recipe should be modified. There are basically two ways to modify it; either by multiplying the number of objects by two or by multiplying the mass by a factor of 2 . This former alternative will be adopted here, as in the original PS work, and in accord with numerical simulations. The mass function is written

$$
N(M)=\sqrt{\frac{2}{\pi}} \frac{\bar{\rho}}{M^{2}} \frac{f_{c}}{\sigma} \frac{d \ln \sigma}{d \ln M} e^{-v^{2} / 2}
$$

with

$$
v=\frac{f_{c}}{\sigma(R)},
$$

where $\sigma$ is the variance of fluctuations within a sphere of radius $R$ containing mass $M=4 \pi / 3 \bar{\rho} R^{3}$.

Another free parameter is the normalization of the spectrum. Here we define $b$ as

$$
\left(\frac{\delta M}{M}\right)_{8 h^{-1} \mathrm{Mpc}}=\frac{1}{b} .
$$

We assume a value of $b=1.7$ which leads to a mean cluster $M / L$ ratio of around $200 h$, in good agreement with observations.

The models we investigate are derived by Evrard \& Henry (1991). They are summarized in Table 1. Model CDMSS employs self-similar scaling in a CDM cosmology. Model CDMP3 has a scaling of $p=3$ in CDM while model XCDM employs an $n=-2$ spectrum and values of $p=11 / 6$ and $s=11 / 4$. Evrard \& Henry found that the latter two models produce luminosity functions in good agreement with local observations (see also the following section 3.2). The latter model has scaling laws consistent with the minimum central entropy for cluster gas, a feature which could be generated by feedback during the galaxy formation era.

According to the scaling in equation (9), clusters of given mass were hotter in the past as $T(z) \propto(1+z)$. However, because of the frequency shift in an expanding universe, the observed temperature remains constant: $T_{\text {obs }}=T(z) /(1+z)=$ $T(0)$. Therefore, a given observed temperature corresponds to a given mass independent of cluster redshift. A temperaturelimited survey of clusters will map clusters in a given mass range whatever their redshift. Since rich clusters are presently rare events (Kaiser 1986; Cole \& Kaiser 1989), their number is expected to decrease rapidly with lookback epoch. Their contribution to the X-ray background in a fixed energy band will decrease rapidly, unless luminosity evolution is extreme.

\section{DIFFUSE X-RAY BACKGROUND SIMULATIONS}

Monte Carlo methods are used to simulate counts of photons incident on the pixels of the Einstein IPC Detector and the ROSAT PSPC detector from galaxy cluster sources. We first generate a sample of clusters in a 10 square degree circular patch of sky as a random realization of the PressSchechter mass function, assigning each cluster a mass, redshift, and position. An arbitrary patch of sky is represented, with no attempt to avoid bright sources. 


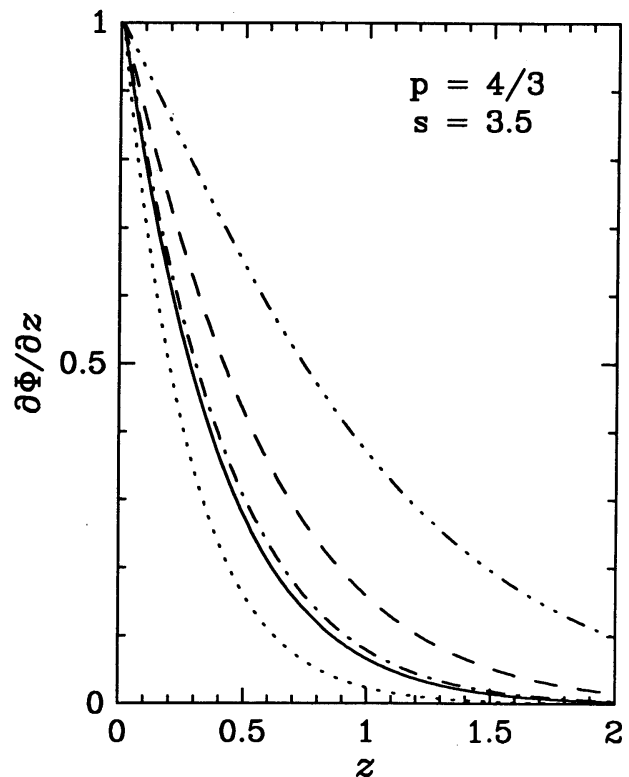

FIG. $1 a$

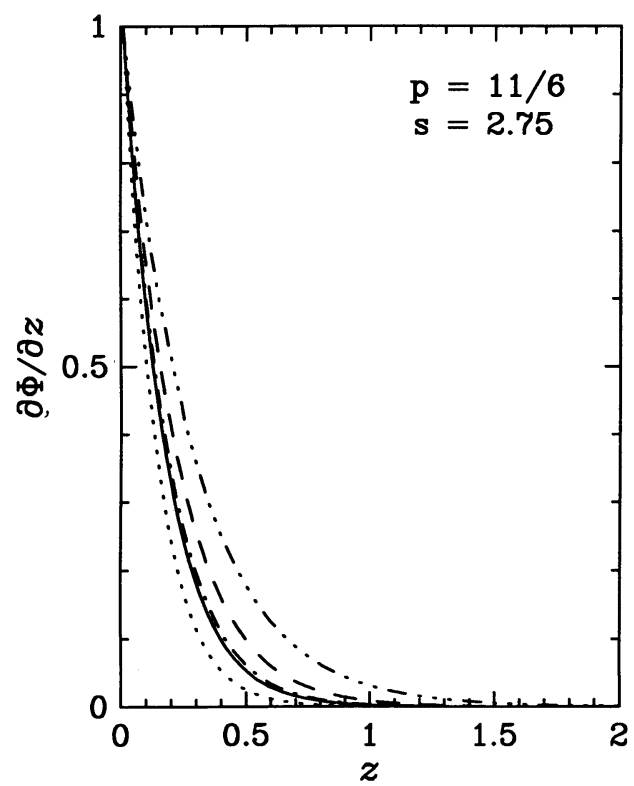

FIG. $1 c$

FIG. 1.-Relative contribution to the predicted flux as a function of redshift. Soft X-ray bands probe deeper in redshift in the self-similar model. Three-dot-dashed lines, ROSAT 0.1-0.4 keV band. Dashed lines, ROSAT $0.5-1 . \mathrm{keV}$ band. Dot-dashed lines, ROSAT 1.-2. $\mathrm{keV}$ band. Solid lines give Einstein 1-3 keV band, and dotted lines HEAO 1 A-1 2-10 keV band. Models shown: (a) CDMSS, (b) CDMP3, (c) XCDM.

This procedure needs a mass cutoff in order to avoid the divergence in the cluster number in the small-mass regime. The mass cutoff was chosen as a function of redshift to correspond to a total flux limit of $10^{-14}$ ergs $\mathrm{cm}^{-2} \mathrm{~s}^{-1}$, ensuring that any cluster that could have been detected by Einstein is included in the sample. As described in the next section, each simulation includes extra photons randomly scattered through the field to make up for the excluded flux.

Each cluster is assigned a temperature and total luminosity

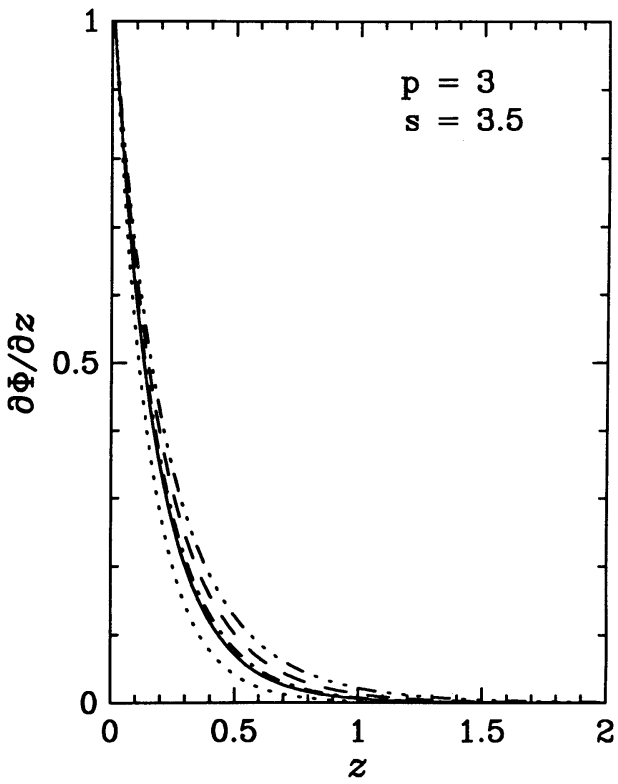

Fig. $1 b$

depending on its mass according to the analytic model outlined above. A log-normal dispersion factor has been included in the $T_{X}-M$ and $L_{X}-M$ relations with amplitudes of $10 \%$ and $50 \%$, respectively.

\subsection{Expected Background Levels}

Figure 1 shows the relative contribution against redshift in different bands: the 1-3 keV Einstein band, the 2-10 keV $H E A O 1$ band and three soft bands in the ROSAT range 1-2 $\mathrm{keV}, 0.5-1 \mathrm{keV}$ and $0.1-0.4 \mathrm{keV}$. Hereafter, we will refer to the latter ROSAT bands as "hard," "soft," and "extremely soft." The response of the PSPC is falling between 0.4 and $0.5 \mathrm{keV}$ and above $2.0 \mathrm{keV}$. This is taken into account in the simulations when we refer to the $0.1-0.5$ and $1 .-2.4 \mathrm{keV}$ band. The set of figures in Figure 1 illustrates the range in redshifts at which groups and clusters contribute to the local XRB. The contribution in the soft bands arises from more distant clusters; this effect is more pronounced in the self-similar model than in models CDMP3 and XCDM.

In Figure 2, we show the cumulative total flux per square degree from the sources in the simulated field as a function of $z$ for the different energy bands. Perhaps the most surprising aspect of the cluster contribution is the fact that despite the sensitive evolution effect, half of the contribution arises from low-redshift objects (typically $z \leq 0.2$ ). This effect is very strong in the CDMP3 and XCDM models, where the contribution in the soft energy band does not probe deeper redshift.

The total contributions in different models are summarized in Table 2. We quote direct measurement of the extragalactic diffuse XRB for the $2-10 \mathrm{keV}$ band only. The levels for the other bands are obtained by extrapolating this flux on the basis of a $40 \mathrm{keV}$ thermal bremsstrahlung distribution (Boldt 1987). This is most likely a lower bound to the extragalactic component lower energies (Wu et al. 1991). In model CDMSS, most of the background at soft energies would be produced by clusters. This is unrealistic as a large fraction of the background at energy around $1 \mathrm{keV}$ is known to arise from discrete sources such as QSOs and AGNs. The soft contribution is higher in the XCDM model compared to the CDMP3 model. 


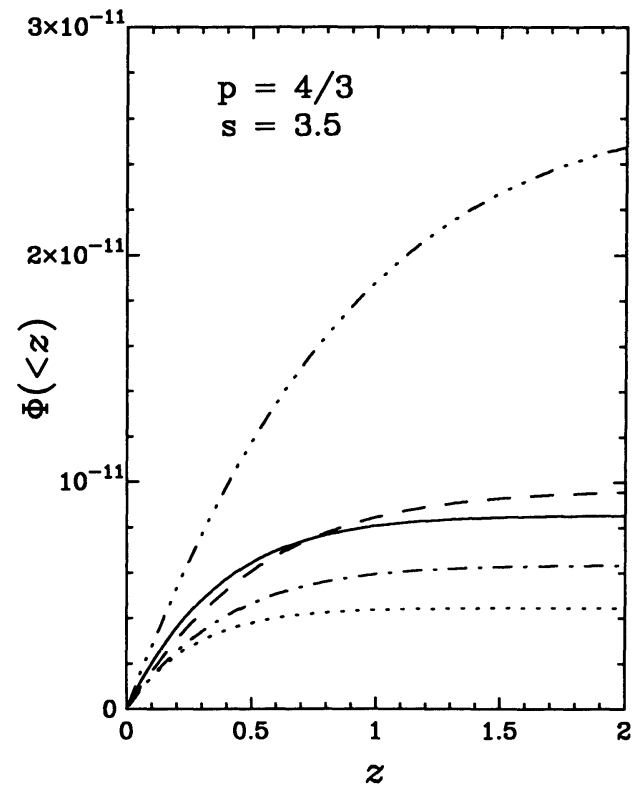

FIG. $2 a$

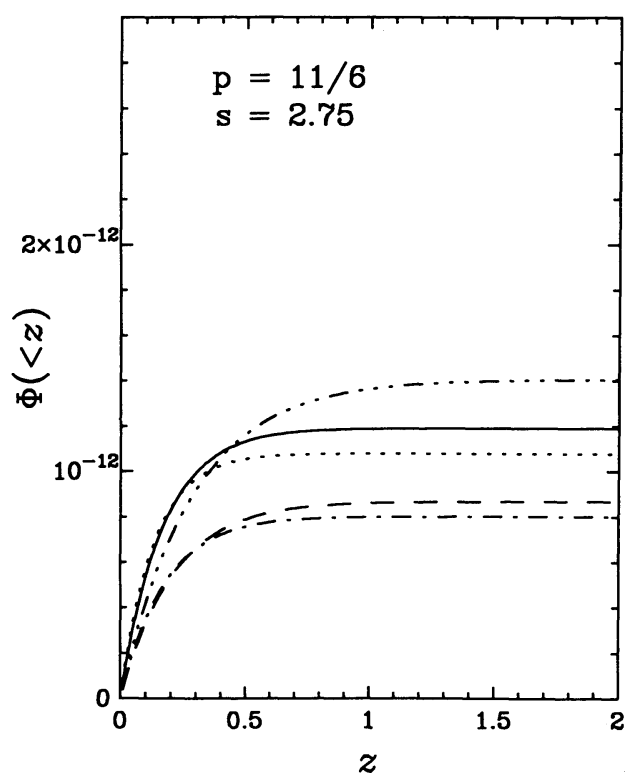

FIG. $2 c$

Fig. 2.-Cumulative total predicted flux per square degree as a function of source redshift. Lines are as in Fig. 1. Most of the contribution clearly comes from relative nearby objects. Models shown: (a) CDMSS, (b) CDMP3, (c) XCDM.

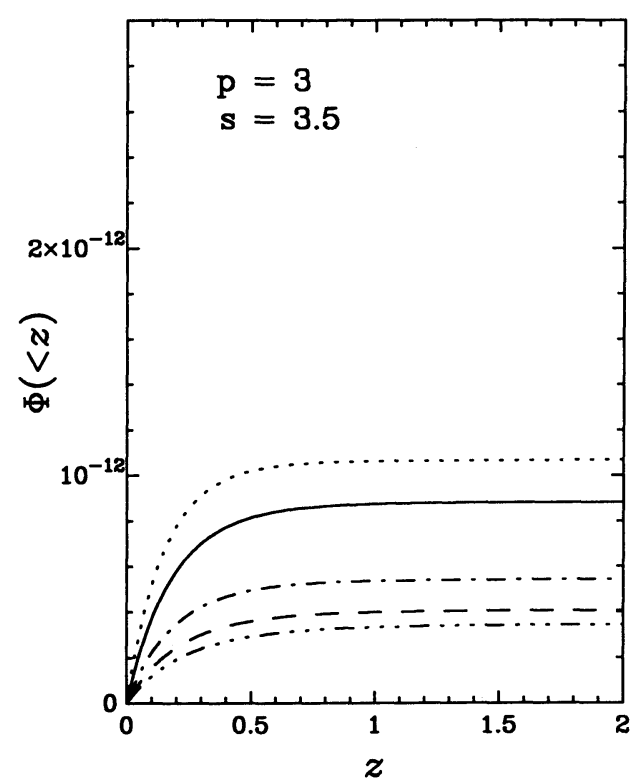

FIG. $2 b$

For the self-similar model, in the $2-10 \mathrm{keV}$ band, we find that the contribution of clusters to the background represents $25 \%$ of the observed intensity, and is slightly sensitive to the value of $p$ : in CDMP3 and XCDM this contribution is still of the order of $8 \%$. This is due to the fact that most of the contribution comes from clusters of mass a few times $10^{15} M_{\odot}$, at which scale our relation for the different models has been normalized. In the softer bands, the contribution of clusters is reduced by an order of magnitude.

In the 1-3 keV Einstein band, we find that the observed flux could be substantially larger than the flux extrapolated from the bremsstrahlung fit. The flux of the observed background in the 1-3 keV band does appear to exceed the fiducial $40 \mathrm{keV}$ bremsstrahlung extrapolation of the hard X-ray background (Wu et al. 1991). This leaves room for the possibility that the cluster contribution represents the dominant fraction of the extragalactic background at soft energies.

\subsection{Luminosity Function and Number Counts}

The value we have obtained for the total flux is quite high compared to some previous estimates of the contribution of clusters. Two effects are responsible for increasing our value for the total contribution: first, the effect of evolution, and, second, the contribution of low-luminosity clusters. This explanation can be confirmed by examination of the luminosity function. Figure 3 illustrates the luminosity function in the different bands. In the self-similar model we find that the number agrees

TABLE 2

FLUX IN DIFFERENT BANDS

\begin{tabular}{cccccc}
\hline Model & $0.1-0.4 \mathrm{keV}$ & $0.5-1 \mathrm{keV}$ & $1-2 \mathrm{keV}$ & $1-3 \mathrm{keV}$ & $2-10 \mathrm{keV}$ \\
\hline CDMSS $\ldots \ldots \ldots \ldots \ldots \ldots$ & 26.2 & 9.66 & 6.35 & 8.56 & 4.46 \\
CDMP3 $\ldots \ldots \ldots \ldots \ldots \ldots$ & 0.34 & 0.41 & 0.54 & 0.88 & 1.07 \\
XCDM $\ldots \ldots \ldots \ldots \ldots \ldots$ & 1.40 & 0.87 & 0.80 & 1.19 & 1.08 \\
Observed $\ldots \ldots \ldots \ldots \ldots$ & $1.75^{\mathrm{a}}$ & $2.0^{\mathrm{a}}$ & $3.3^{\mathrm{a}}$ & $5.9^{\mathrm{a}}$ & 15.8 \\
\hline
\end{tabular}

NoTE.-The fluxes are given in unit of $10^{-12} \mathrm{ergs} \mathrm{s}^{-1} \mathrm{~cm}^{-2} \mathrm{deg}^{-2}$.

${ }^{a}$ based on the thermal fit in the $3-50 \mathrm{keV}$ range. The actual value is probably higher. 


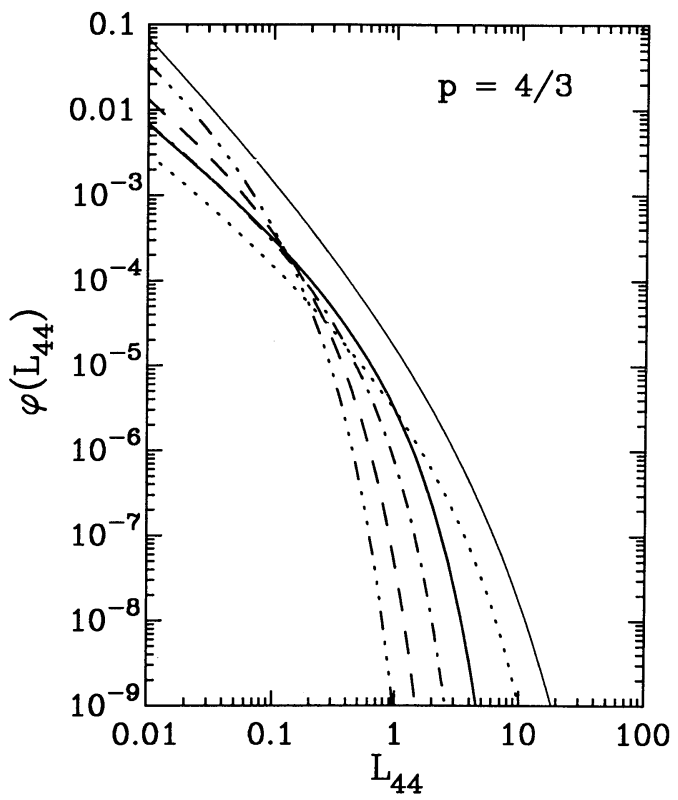

FIG. $3 a$

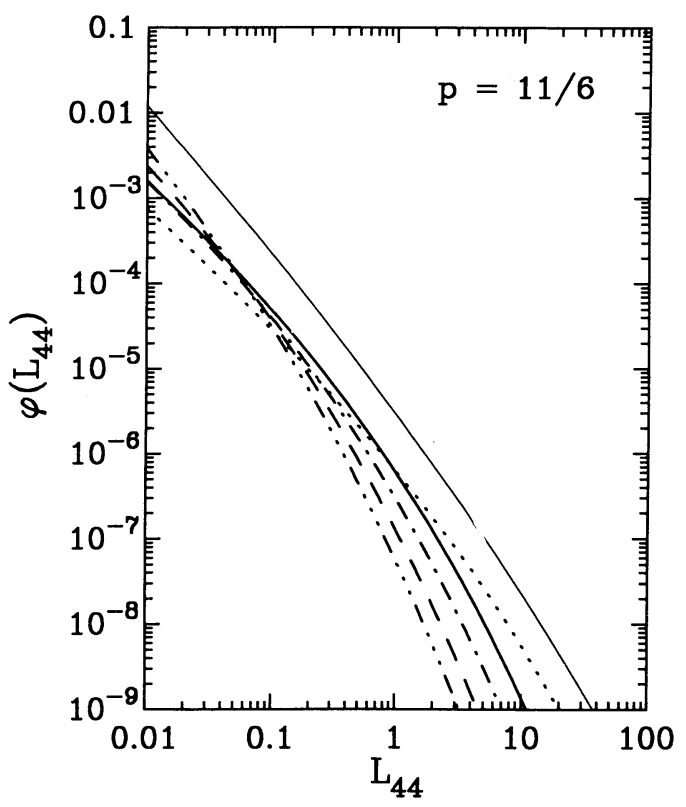

Fig. $3 c$

Fig. 3.-Luminosity functions predicted for the different models. The number of clusters is given per $\mathrm{Mpc}^{-3}$ per decade of luminosity in units of $10^{44}$ ergs s${ }^{-1}$ vs. $\log _{10}$ of luminosity in units of $10^{44} \mathrm{ergs} \mathrm{s}^{-1}$. This is to be compared with the observed luminosity function: $2.2 \times 10^{-7} L_{44}^{-2.17}$ (Edge et al. 1990). The lines for the different bands are as in Fig. 1. The thin solid line corresponds to the bolometric luminosity function. Models shown: (a) CDMSS, (b) CDMP3, (c) XCDM.

with observation of clusters with $L_{X} \sim 10^{45} \mathrm{ergs} \mathrm{s}^{-1}$, but we find an excess of clusters at $L_{X} \sim 10^{44} \mathrm{erg} \mathrm{s}^{-1}$, as compared to the X-ray luminosity derived from $H E A O 1$ (McKee et al. 1980; Piccinotti et al. 1982; Edge et al. 1990). A cautious stance is appropriate, however, with regard to the actual detectability of these clusters. They mostly have low temperatures, in the range of $1-3 \mathrm{keV}$, and they might have been missed for this reason. Furthermore, clusters are extended sources which may

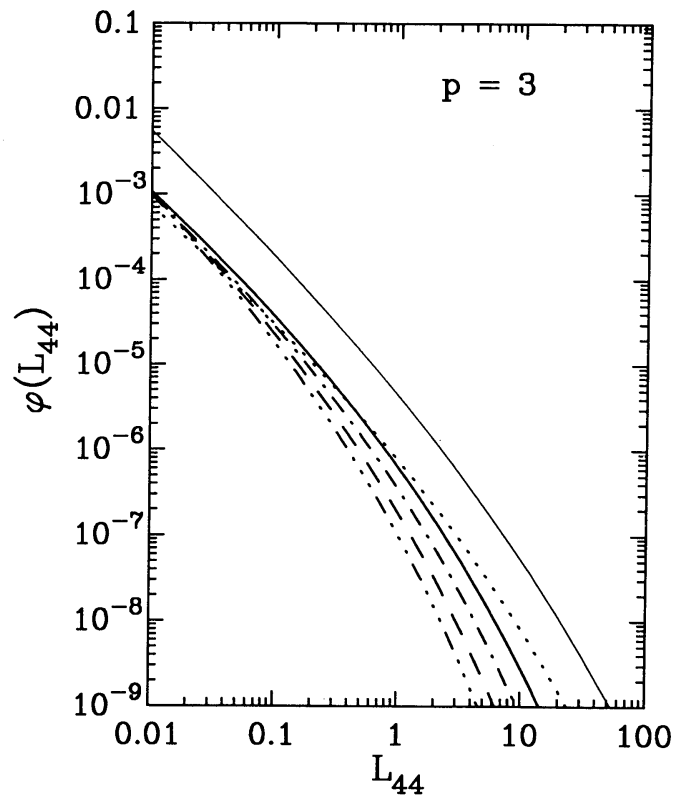

FIG. $3 b$

sometimes elude point-source detection algorithms. Only by breaking the self-similar assumption are we able to find models whose results are consistent with the observed luminosity function. In CDM models we find that a value of $p$ as high as 3 is necessary for consistency. In power-law models, $n=-2$ with $p=11 / 6$ leads to similar results (Evrard \& Henry 1991).

The analytic scaling models incorporate a substantial degree of luminosity evolution, clusters of a given mass being intrinsically brighter in the past by a factor $(1+z)^{2.75-3.5}$. However, the number density of the hottest clusters falls exponentially at higher $z$, effectively countering the luminosity increase. Evolutionary effects will therefore not be critical to our discussion, since the dominant portion of the total background comes from redshifts $z \lesssim 0.5$ for the Einstein and HEAO 1 bands and $z \lesssim 1$ for the $R O S A T$ band. The dominant source of uncertainty in the problem is the $L_{X}-M$ relation, which actually depends on the $\mathrm{X}$-ray core properties.

Figure 4 shows the relative contribution to the background at low redshift as a function of cluster mass (this is the mass embedded in a sphere for which the total density contrast is 170). As expected, it appears that the contribution of typical Abell clusters is essentially the same in the different bands while the contribution in softer bands comes from clusters of lower mass, corresponding to rich groups. In the self-similar model, the main contribution to the $1-3 \mathrm{keV}$ band comes from clusters of mass close to $2 \times 10^{14} M_{\odot}$ but a substantial contribution in the ROSAT bands could come from low-mass systems. This does depend, however, on the specific model; this contribution is almost suppressed in the other models presented here. At higher redshift, the position of the maximum contribution moves toward smaller mass clusters; however, the change between $z=0$ and $z=1$ is small.

In Figure 5 we show a $\log N-\log (>S)$ plot for the different cases and for different energy bands. This figure is based on assuming clusters are point sources, a procedure which will overestimate the flux expected in a detect cell of $\sim 2^{\prime}$ size for nearby clusters. Consequently, the slope is flatter at high flux than that seen in the Extended Medium Sensitivity Survey 


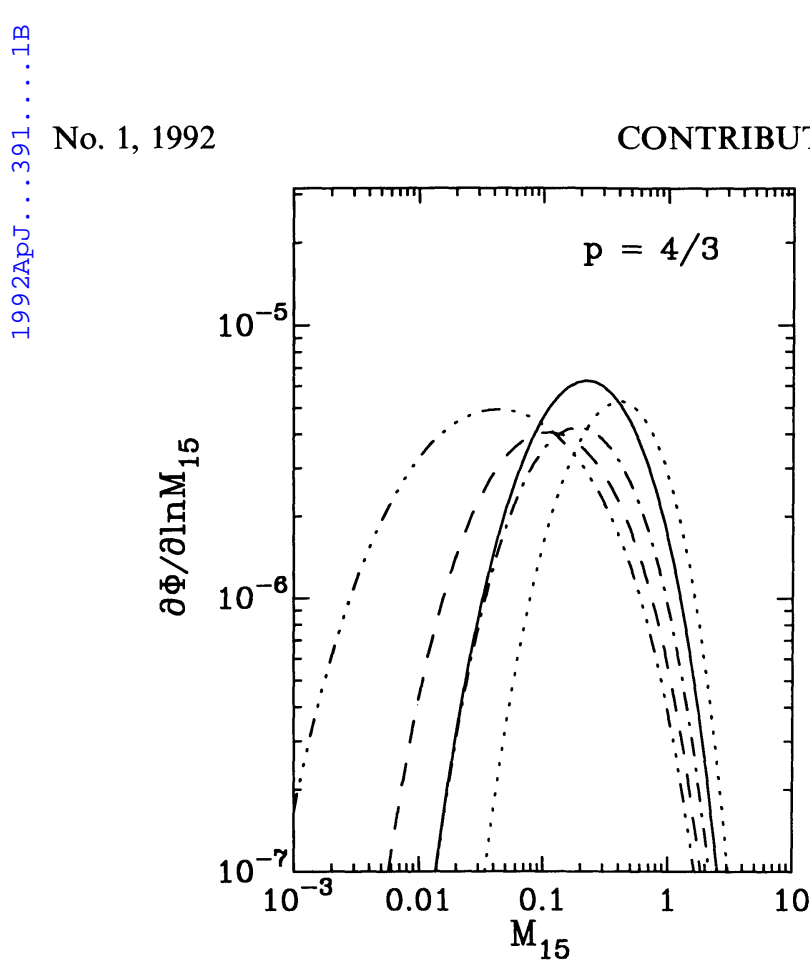

FIG. $4 a$

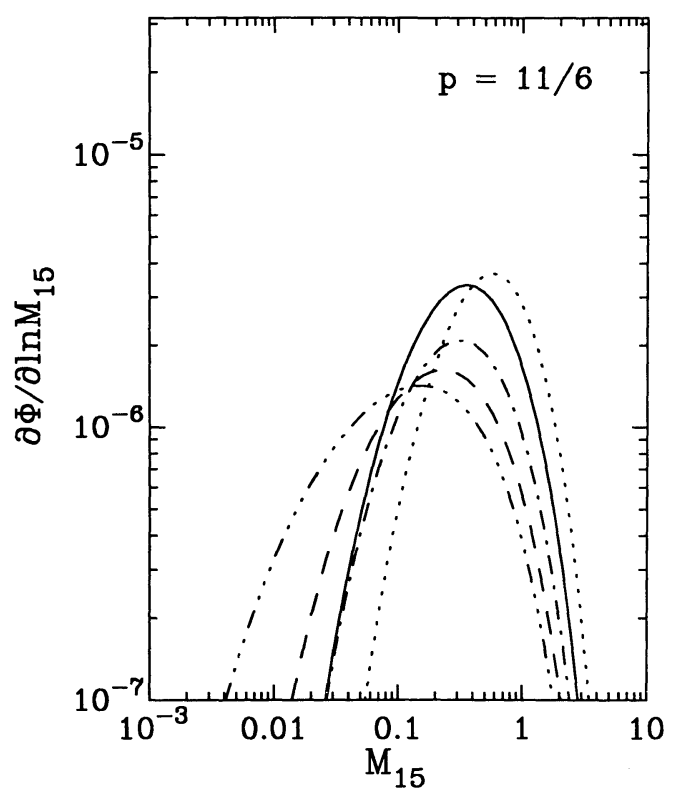

FIG. $4 c$

FIG. 4.- Relative contribution to the background as a function of the mass at $z=0$. The plotted quantity is in $10^{44} \mathrm{ergs} \mathrm{s}^{-1} \mathrm{Mpc}^{3}$. The maximum of such a curve indicates the mass of clusters which are the dominant contribution in the given energy band. The lines for the different bands are as in Fig. 1. Models shown: (a) CDMSS, (b) CDMP3, (c) XCDM.

(Gioia et al. 1990). We expect $\sim 10$ clusters at the flux limit of presently available samples $\left(\sim 10^{-14} \mathrm{ergs} \mathrm{s}^{-1} \mathrm{~cm}^{-2}\right)$. The first results from deep imaging detected an extended "blotch" of soft, diffuse emission which is now known to arise from thermal emission of gas in a nearby small group of galaxies.

\subsection{Detector Images}

Images of the Monte Carlo cluster fields can be produced with one additional assumption fixing the scaling of core radius with mass. The core radius is critical in determining the

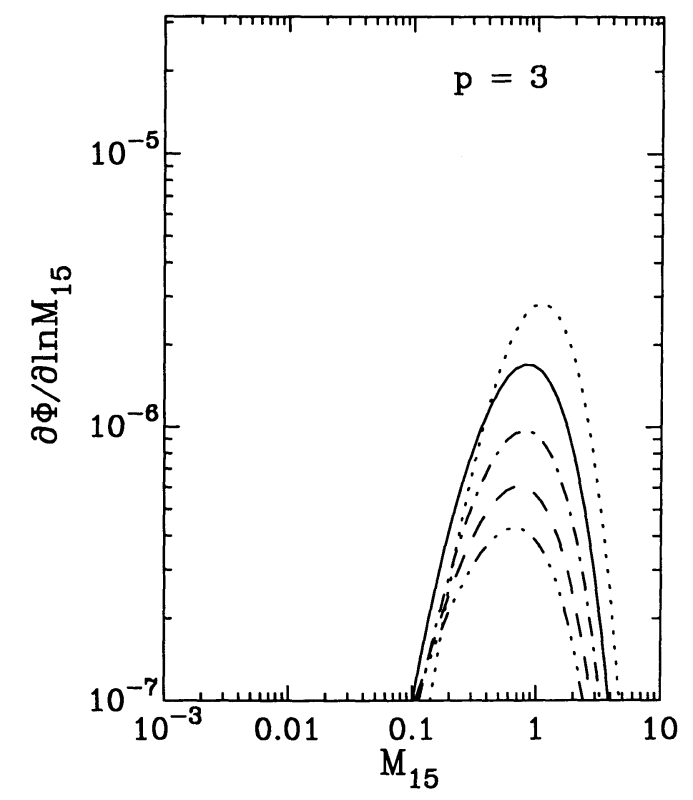

FIG. $4 b$

central surface brightness and hence the ease of detection above a prevailing threshold. For the self-similar model, the core radius is given by $R_{c}=300 M_{15}^{1 / 3} \mathrm{kpc}$. For both models CDMP3 and XCDM, we take the core radius to be constant at $300 \mathrm{kpc}$. Since observed core radii span a reasonable range, we include a log-normal dispersion with an amplitude estimated from the observed dispersion $\Delta R_{c} / R_{c}=0.5$.

The total flux from each cluster is distributed across the Einstein and ROSAT bands assuming bremsstrahlung emission at the given apparent temperature of each source. Einstein fluxes are converted to photon counts using a constant conversion factor of one photon per $2.5 \times 10^{-11} \mathrm{ergs} \mathrm{cm}^{-2}$ (Giacconi et al. 1979). ROSAT fluxes are converted to photon counts by linear interpolation of Figure 10.3 of Appendix $F$ of the ROSAT specifications which shows net counts per unit flux as a function of source temperature. A column density of $10^{20}$ $\mathrm{H}$-atoms $\mathrm{cm}^{-2}$ is assumed. This gives on average one photon per $0.866 \times 10^{-11} \mathrm{ergs} \mathrm{cm}^{-2}$. Lower detector efficiencies at lower temperatures roughly compensate the higher numbers of photons per unit flux at lower temperatures to give the flat conversion profile for both detectors over the relevant source temperature range.

A Poisson-distributed random total count of photons is generated by rounding a random normal deviate with mean and variance both equal to the sum of the mean counts for all the sources times the chosen exposure time. A set of sources is excluded on the basis of the expected number of photons whose true positions (before blurring by the point response function of the detector) would fall within the detector window. The excluded sources are chosen to account in toto for less than one photon per hour. For the Einstein simulations, this exclusion serves merely to enhance computing efficiency. For the ROSAT simulations, it serves in place of imposing a cutoff on the point-response function of high off-axis angles. The point response function, given below, has a 2.34 power dependence of standard deviation on off-axis angle which would otherwise imply a nonzero contribution from rings of sky at higher and higher off-axis angle.

Each simulated photon is assigned to one of the non- 


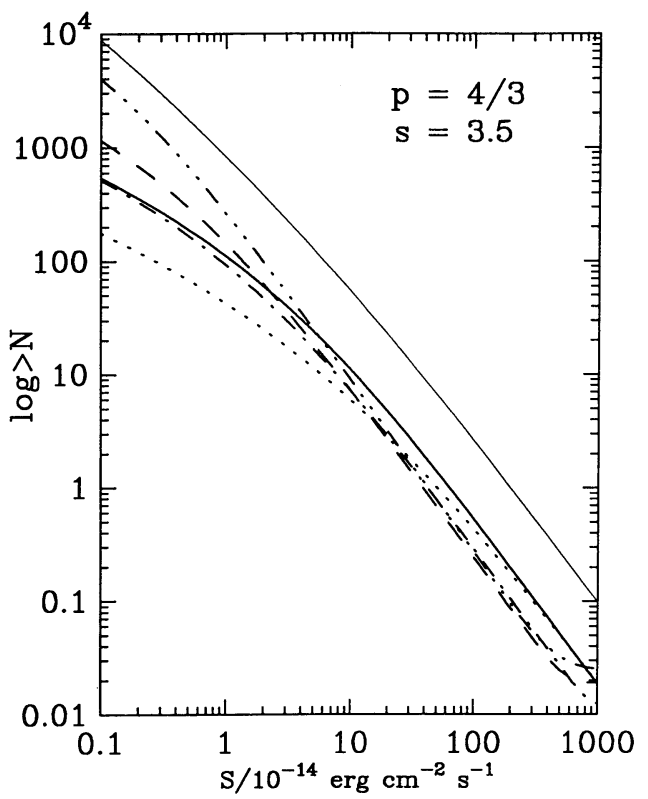

FIG. $5 a$

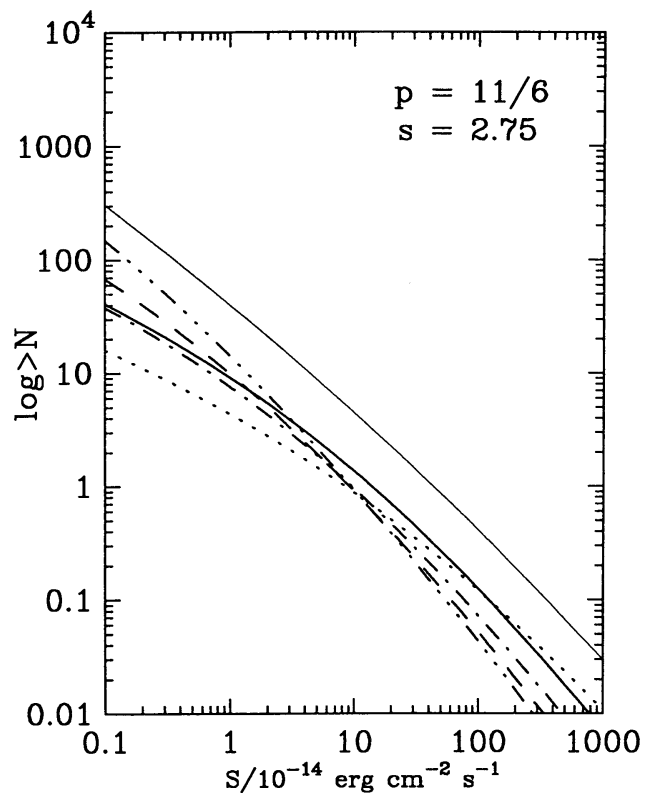

Fig. $5 c$

Fig. 5.- $\log N$ vs. $\log (>S)$ of the counts of sources per square degree above each given level of $\log _{10}$ flux in unit of $10^{-14} \mathrm{ergs} \mathrm{s}^{-1} \mathrm{~cm}^{-2}$. The lines are as in Fig. 3. Models shown (a) CDMSS, (b) CDMP3, (c) XCDM.

excluded sources by drawing a uniform random number and comparing it against a subdivision of the unit interval into pieces proportional to each source's share in the mean total count. The sky position from which the photon is taken to originate is isotropically distributed about its source center with distance given in terms of a uniform random number $u$ on the unit interval by its cluster core radius times

$$
\sqrt{-1+u^{-2}} \text {. }
$$

This means that the photons are distributed according to the

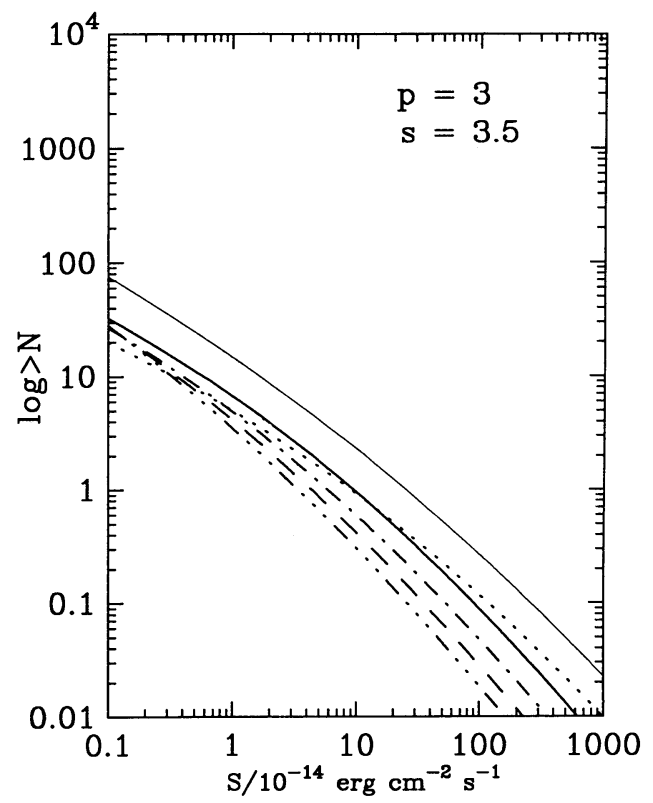

Fig. $5 b$

projected luminosity profile

$$
S(\theta) \propto \frac{1}{1+\left(\theta / \theta_{c}\right)^{1.5}}
$$

corresponding to the spatial density profile of equation (6) with $\beta=\frac{2}{3}$. An isotropic bivariate-normal error term is then added to this original position. For Einstein a constant standard deviation of 0.5 is assumed for the point response function. For ROSAT the standard deviation in arcminutes is given as a function of original off-axis angle $\theta$ and photon energy $E$ (uniformly distributed across the band) by the point response function

$$
(1 / 60) \sqrt{92.16 / E+2.25+\left(1.3+0.02 \theta^{2.34}\right)^{2}} .
$$

The photons are collected in bins corresponding to disjoint square pixels on the detector. For Einstein, pixels are 64" on a side and range between plus and minus $16^{\prime}$ on either side of the detector center. For ROSAT, pixels are $30^{\prime \prime}$ on a side and the same square in the center of the circular detector of $1^{\circ}$ radius is used. Outside this square, the point response function largely blurs the sources.

To account for the faint sources excluded from the mass function, as discussed above, a Poisson scatter of extra photons is superposed on the cluster contributions. For the Einstein band, the extra photon counts average $23 \%, 3.5 \%$, and $5 \%$ of the cluster contributions for the models with $p$ equal to $4 / 3,3$, and $11 / 6$, respectively. For the ROSAT bands, they average $81 \%, 9 \%$, and $32 \%$. An additional component of background noise is also superposed. For Einstein, this represents a charged particle background of $3.4 \times 10^{-4}$ counts s $\mathrm{s}^{-1} \operatorname{arcmin}^{-2}$. For ROSAT, the background from charged particle noise can be safely neglected. The instrumental background of ROSAT, the primary limitation for source detection, will come from the unresolved component of the background. We have estimated the amplitude of the background below $3 \mathrm{keV}$ by assuming the spectral shape to be $E^{-1.3}$ and by assuming the intensity of the diffuse component to be $55 \%$ of the level observed at $3 \mathrm{keV}$ by Wu et al. (1991). This results in Poisson count rates of 7.6, 1.7, 

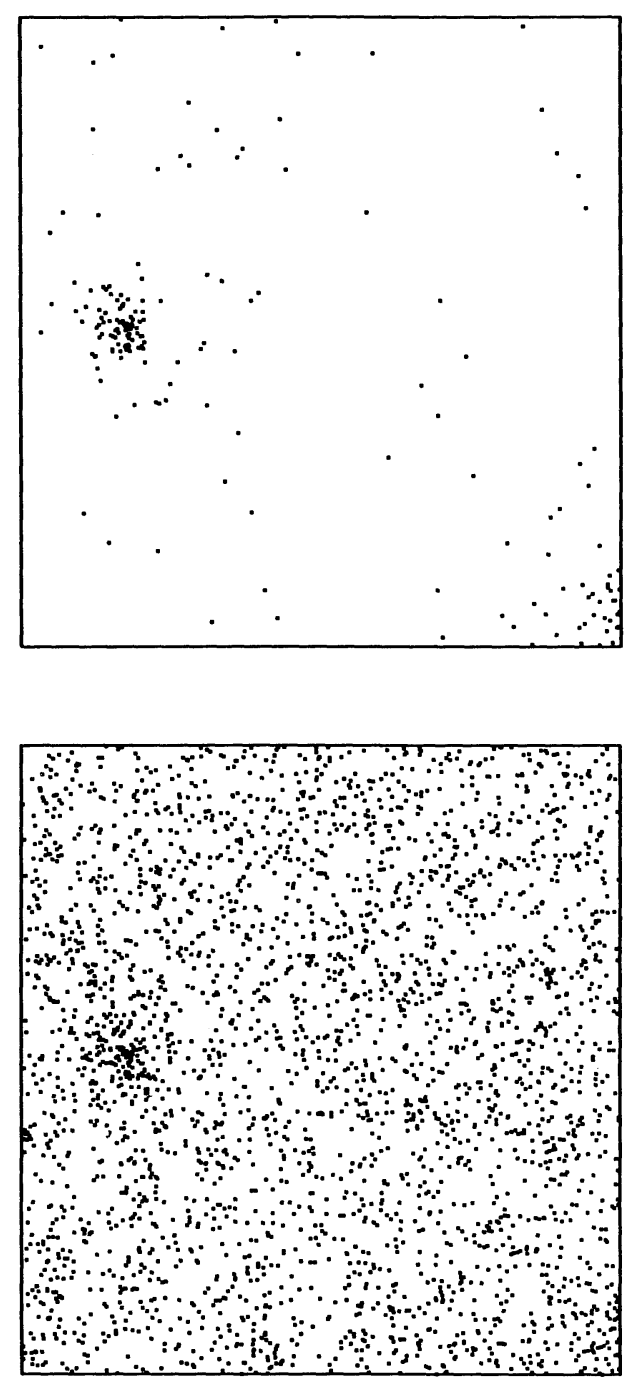

FIG. 6.-Photon positions from $5 \mathrm{hr}$ exposure in simulated ROSAT field in the soft $0.5-1 \mathrm{keV}$ band for model CDMP3. Two panels show photons from cluster sources only (top) and source plus background (bottom).

and $1.6 \times 10^{-4}$ counts $\mathrm{s}^{-1} \operatorname{arcmin}^{-2}$ for the $0.1-0.5,0.5-1$, and $1-2.4 \mathrm{keV}$ bands, respectively.

Figure 6 shows simulated ROSAT sky maps for model CDMP3. Each dot represents a single photon in a $5 \mathrm{hr}$ exposure in the soft $0.5-1 \mathrm{keV}$ band. The top panel shows photons from cluster sources, the bottom shows source plus background photons in the 30 arcmin square. Plots for the XCDM model are very similar as the same catalog of clusters underlies all of our simulated fields. The visual effect in Figure 6 is dominated by a single bright extended cluster at center left and, to a lesser extent, by a bright cluster centered off the detector at lower right. Away from the brightest sources the eye would have some difficulty in telling this pattern apart from a random Poissonian background.

The upper panel of Figure 7 shows a perspective plot for the photons in each pixel for the $5 \mathrm{hr}$ simulation of galaxy cluster contributions for the Einstein detector for the self-similar model. For the lower panel, the noise contribution has been superposed. Except for the two dominant sources, the source contributions are only distinguished from the noise with difficulty. Without the noise, the 900 pixels have a mean count of
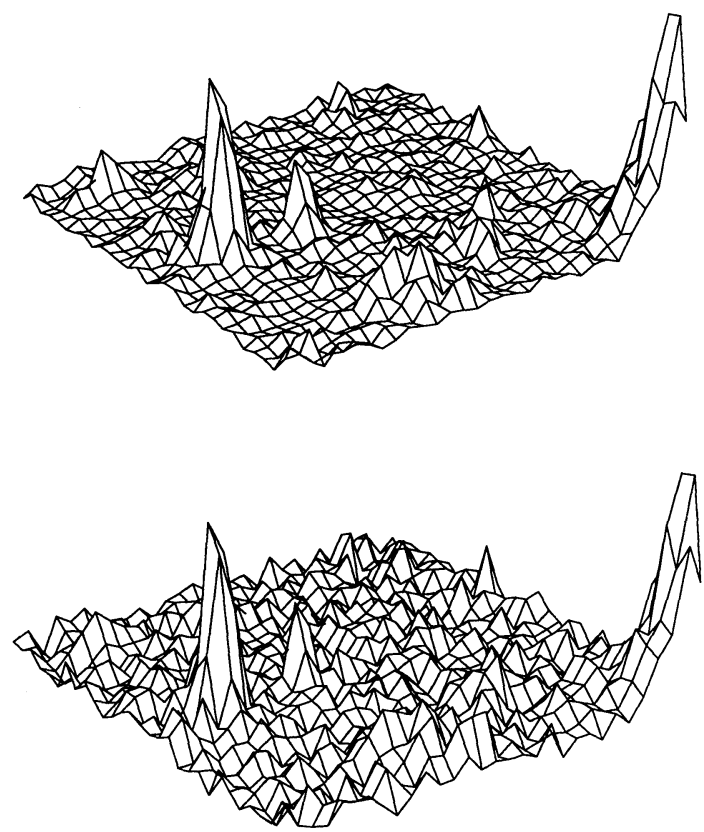

FIG. 7.- Sources vs. sources plus noise, perspective plots of photon counts in each pixel of a simulated detector grid for Einstein for the CDMSS model. The top panel includes photons only from sources, the bottom one photons from sources plus background.

3.37 and a variance of 34.4 . With noise included, the mean is 10.37 and the variance is 40.2 .

The first constraint that can be placed on the models comes from the source counts in the Einstein deep survey (Giacconi et al. 1979). Above a faint-source detection limit of $2.6 \times 10^{-14}$ ergs $s^{-1}, \sim 20$ sources per square degree were found. This limit is uncertain as recent reanalysis (Hamilton, Helfand, \& Wu 1991) has failed to detect sources at this sensitivity level. However, we shall use the number counts from the Einstein deep survey as an upper limit. These sources had a $S / N$ ratio of 5 in the $\sim 5 \mathrm{hr}$ deep field exposures. Optical and radio followup observations have shown that many of the detected sources are AGN and quasars, but the statistics are not sufficient to rule out clusters at a level up to about 10 sources per square degree, or up to about three sources on the 0.25 square-degree detector field. We adopt this as a working upper limit.

Table 3 shows predictions pertaining to numbers of detectable sources in a square area one-quarter square degree centered on each detector center. For this table, ROSAT pixels have been combined to $1^{\prime}$ squares to give a 30 by 30 array of

TABLE 3

Detectable Sources in Simulations

\begin{tabular}{clrc}
\hline \hline $\begin{array}{c}\text { Detector } \\
(1)\end{array}$ & $\begin{array}{c}\text { Model } \\
(2)\end{array}$ & $\begin{array}{c}N_{\text {pix }}{ }^{\mathrm{a}} \\
(3)\end{array}$ & $\begin{array}{c}N_{\text {reg }} \mathrm{b} \\
(4)\end{array}$ \\
\hline Einstein $\ldots \ldots \ldots \ldots \ldots$ & CDMSS & 35 & 8 \\
& CDMP3 & 7 & 1 \\
& XCDM & 12 & 1 \\
RSAT $^{\mathrm{c}} \ldots \ldots \ldots \ldots$ & CDMSS & 526 & $(>20)$ \\
& CDMP3 & 20 & 2 \\
& XCDM & 38 & 4 \\
\hline
\end{tabular}

${ }^{\text {a }}$ Number of pixels above $5 \sigma$ threshold.

${ }^{b}$ Number of distinct regions above threshold.

c $0.5-2.4 \mathrm{keV}$ band. 
pixels comparable to the configuration for the Einstein simulations. For both satellites, a constant detector threshold is defined, equal to 5 background standard deviations above the mean background level. For Einstein, assuming a Poissondistributed background noise of $3.4 \times 10^{-4}$ particles per second per square arcminute, the threshold equals 18.49 particles per pixel in a $5 \mathrm{hr}$ exposure. Thus $5 \sigma$ corresponds closely to the average number of photons from a source at the Einstein survey limit, which is 18.72 .

The upper half of Table 3 pertains to the Einstein detector with the 1-3 keV band. The lower half pertains to ROSAT, with the full $0.5-2.4 \mathrm{keV}$ band. The three rows within each panel correspond to our three models. Column (2) gives the model. Column (3) shows the number of pixels whose total photon count (source plus noise) exceeds the constant threshold level. Because our sources are extended, several adjacent pixels above threshold may represent a single source. Column (4) shows the number of disjoint regions above threshold in the square detect region. This column is a better measure of the number of sources likely to be detected.

The self-similar prescription of clusters in the first column gives a count for Einstein far in excess of the three or so discrete sources anticipated in a 0.25 square degree field from the Giacconi et al. (1979) analysis. Model CDMP3 and the power-law model XCDM have only a single patch over threshold in this simulation and are safely within the adopted deep survey limit. The ROSAT entries show a huge increase in above-threshold patches for the self-similar model, but only a modest increase for the two more tenable models, despite the enhanced ROSAT sensitivity

Figure 8 dramatizes the differences between simulated model predictions for the ROSAT bands. These perspective plots show source photons without the background for each pixel for the three models. The positions, masses, and the relative radii of the clusters themselves are the same in all the panels, but the number and spread of photons differ according to the model chosen. For completeness, we include perspective plots for the ROSAT extremely soft $0.1-0.4 \mathrm{keV}$ band, where we expect foreground galactic emission and absorption to dominate the ROSAT maps. In this extreme-soft band, the 15319 photons for the self-similar model contrast vividly with the 486 photons from model CDMP3 and the 1758 photons from the model XCDM. In the soft band the corresponding total counts are 8066,521 , and 1282 , and in the hard band they are 7653 , 987, and 2075.

The correlation function of the diffuse XRB was first estimated by Barcons \& Fabian (1989), who argued that clusters would produce excessive correlations if they contribute significantly to the 1-3 keV XRB. However, their models based on point sources did not take into account the fact that lowredshift $(z \lesssim 1)$ cluster emission is extended. Soltan (1991) reanalyzed the Einstein data and concluded the signal found by Barcons \& Fabian was due to the instrumental response, rather than due to real clustering of the photons. He derived a much more stringent limit: $\omega \leq 3 \times 10^{-3}$ between $2^{\prime}$ and $5^{\prime}$, a result consistent with $R O S A T$ preliminary analysis. Since our maps naturally take into account the extended nature of the $\mathrm{X}$-ray emission, it is interesting to compute the correlation function. Under the assumption that detector noise is spatially uncorrelated, Barcons \& Fabian multiplied by a factor $\sim 20$ the correlation ratio (the ratio of covariance to square mean) for their total count ratio to obtain a measure applying to signal alone rather than signal plus noise. They quote an upper
TABLE 4

Simulated Correlations for Einstein IMAGes

\begin{tabular}{ccccr}
\hline $\begin{array}{c}\text { Model } \\
(1)\end{array}$ & $\begin{array}{c}C_{1}{ }^{\mathrm{a}} \\
(2)\end{array}$ & $\begin{array}{c}C_{2}{ }^{\mathrm{b}} \\
(3)\end{array}$ & $\begin{array}{c}\operatorname{Exp}^{\mathrm{c}} \\
(4)\end{array}$ & \multicolumn{1}{c}{$\begin{array}{c}\bar{N}^{\mathrm{d}} \\
(5)\end{array}$} \\
\hline CDMSS $\ldots \ldots \ldots \ldots \ldots$ & 0.00087 & 0.025 & 20 & 39.5 \\
CDMP3 $\ldots \ldots \ldots \ldots \ldots$ & 0.00060 & 0.065 & 100 & 144.1 \\
XCDM $\ldots \ldots \ldots \ldots \ldots$ & 0.00103 & 0.102 & 100 & 137.3 \\
\hline
\end{tabular}

a Correlation measure at 5 '.

b Statistical correlation measure.

c Hours exposure

${ }^{d}$ Mean counts per included pixel.

limit on this transformed ratio of 0.09 . The corresponding limit for a ratio based on signal plus noise is 0.004 . We adopt this value to compare with our simulated maps.

Predicted correlation indicators from our simulations for comparison with the Barcons-Fabian upper limit are shown in Table 4 . The sampling variability from $5 \mathrm{hr}$ exposures is too great for stable estimates, so these values are based on $20 \mathrm{hr}$ exposures for the self-similar model and $100 \mathrm{hr}$ exposures for the two remaining models. All apply to the Einstein band with pixels $64^{\prime \prime}$ on a side at a separation of $5^{\prime}$. All pixels more than five noise standard deviations above the mean noise level have been excluded in this calculation, a rough approximation to Barcons \& Fabian's exclusion procedure. (Column [5] of the table shows the mean count among nonexcluded pixels.) All values for the Barcons \& Fabian ratio fall below their observational limit.

Table 4 also shows for comparison values of the coefficient used in the statistical literature, the ratio of covariance to variance. These too show higher values for the XCDM model. For $R O S A T$, a $100 \mathrm{hr}$ simulation for the CDMP3 model for the soft band produced a correlation of 0.00098 , still below the upper limit derived by Soltan between $2^{\prime}$ and $5^{\prime}$. The exact amplitude of the correlation function due to clusters can be estimated but there are uncertainties that limit the application of this constraint: the amplitude depends on the exact properties of the core radius, which are very uncertain, as we have already pointed out. In addition, the amplitude and the intensity of the background are not yet well known in the soft band and an uncertainty of $50 \%$ still remains at $2 \mathrm{keV}$. This situation should improve rapidly. The constraints on the models are stronger at $2 \mathrm{keV}$ where the relative contribution of clusters is the largest and represents between $15 \%$ and $25 \%$ of the unresolved component. In Table 4, we have assumed that the spectrum measured by $R O S A T\left(\propto E^{-1.3}\right)$ holds up to $3 \mathrm{keV}$, and we have removed $55 \%$ in this band, which we attribute to known sources.

On the basis of these results, it does not seem as if correlation analysis rules out a significant contribution to the XRB from clusters at $1-3 \mathrm{keV}$. It furthermore appears that correlation analysis with long exposures might be useful for distinguishing between models that meet the discrete-source observational constraints.

Figure 9 presents histograms of the number of pixels out of 900 with zero, one, two, etc., photons in the Einstein simulations. As before, the three rows correspond to our three cluster models. The left-hand panels show cluster contributions only; the right-hand panels show the same models with noise superposed on signal. The dotted lines show the number of pixels in which source count exceeds noise count at each value of total 

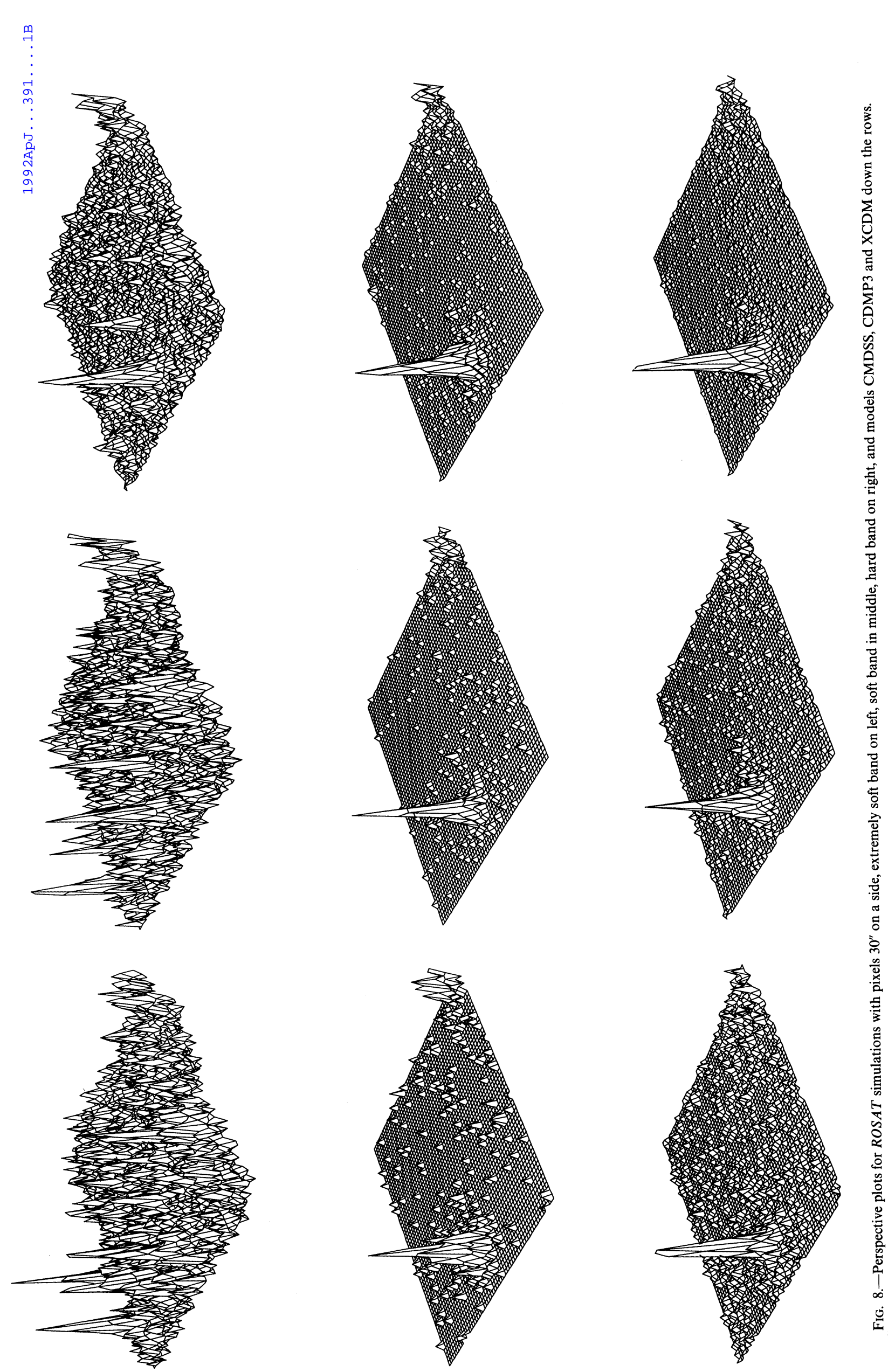

(C) American Astronomical Society - Provided by the NASA Astrophysics Data System 

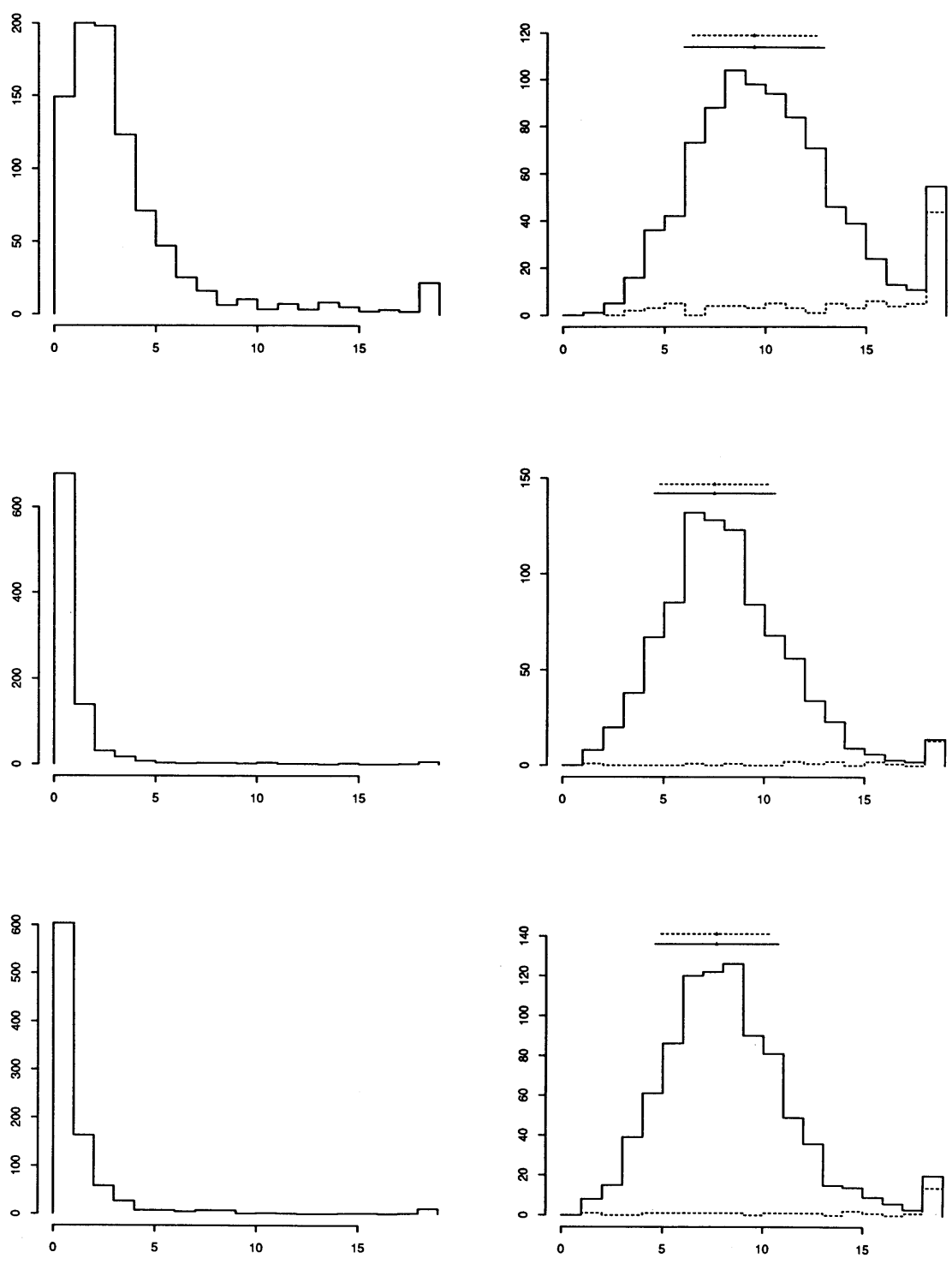

FIG. 9.-Histograms of simulated Einstein pixel counts. Number of pixels with 0, 1, 2, etc., photons for CDMSS at top, CDMP3 in middle, and XCDM at bottom. At left are counts for source photons alone; at right are counts for source photons plus noise. Dotted line at right shows number of pixels where source count exceeds noise count. Solid bar above histogram shows $1 \sigma$ interval around trimmed mean; dotted bar shows the corresponding interval if the variance were set equal to the trimmed mean. Right-most cell is for 18 and above.

photon count. In each histogram, the final interval is an openended interval including all cells with 18 or more photons.

The right-hand panels of Figure 9 are meant to be comparable to Figure 1 of Hamilton \& Helfand (1987). The contributions from clusters alone on the left are much more highly skewed than Poisson counts. When noise is superposed, on the right, the general appearance is more nearly Poissonian, but the spread is slightly greater than Poisson statistics would predict. Deleting all pixels above the threshold used for Table 3 , we have calculated means and variances of the counts pixel by pixel, and $1 \sigma$ intervals above and below the mean are indicated above the histograms on the right with solid lines. The dotted lines give the corresponding intervals for variances set equal to sample means as would occur if the distributions were actually Poissonian.

The excess above unity of the ratio of variance to mean after deleting above-threshold pixels is a common measure of "granularity." For their Figure 1, Hamilton \& Helfand (1987) granularity, is 0.16 . Values predicted from our simulations are shown in Table 5. Column (2) shows the model and column (3) the simulated granularity. Our deletion criterion is a threshold of 5 noise standard deviations above the mean noise level. This criterion is a rough approximation to Hamilton \& Helfand's more complex deletion procedure. Our granularities for the Einstein band and pixel configurations from our three models are $0.277,0.199$, and 0.234 . These are all slightly greater than Hamilton \& Helfand's granularity, but not enough to constrain the models.

Our simulated granularities for $R O S A T$ are rather unstable. The smaller pixels exacerbate a problem inherent in the conventional measure of granularity. Statistically speaking, basing a measure of granularity on the sample variance has some undesirable consequences. The squaring of deviations from the mean in the computation of variances makes the measure most sensitive to the highest photon counts included and therefore most sensitive to the threshold above which counts are 
TABLE 5

Simulated GranUlaRITIES

\begin{tabular}{|c|c|c|c|c|c|c|c|}
\hline $\begin{array}{l}\text { Detector } \\
\text { (1) }\end{array}$ & $\begin{array}{l}\text { Model } \\
\text { (2) }\end{array}$ & $\begin{array}{c}\text { Granularity } \\
\text { (3) }\end{array}$ & $\begin{array}{l}W_{\alpha} \\
(4)\end{array}$ & $\begin{array}{c}\text { Mean } \\
(5)\end{array}$ & $\begin{array}{l}\text { Variance } \\
(6)\end{array}$ & $\begin{array}{c}\text { Trimmed } \\
\text { (7) }\end{array}$ & $\begin{array}{c}\text { Mean } \\
(8)\end{array}$ \\
\hline \multirow[t]{3}{*}{ Einstein } & CDMSS & 0.28 & 2.18 & 10.37 & 40.2 & 3.88 & $\ldots$ \\
\hline & CDMP3 & 0.20 & 0.67 & 7.76 & 18.5 & 2.38 & $\ldots$ \\
\hline & XCDM & 0.23 & 0.85 & 8.20 & 36.9 & 4.49 & $\ldots$ \\
\hline \multirow{6}{*}{$\begin{aligned} \text { ROSAT: } & \text { soft }^{\mathrm{a}} \ldots \ldots \ldots \ldots \ldots \\
& \text { hard }^{\mathrm{b}} \ldots \ldots \ldots \ldots \ldots \ldots \\
& \text { full }^{\mathrm{c}} \ldots \ldots \ldots \ldots \ldots \ldots \\
& \text { soft } \ldots \ldots \ldots \ldots \ldots \ldots \\
& \text { hard } \ldots \ldots \ldots \ldots \ldots\end{aligned}$} & CDMSS & $\ldots$ & -0.14 & 0.32 & 3.01 & 5.92 & 2.37 \\
\hline & CDMSS & $\cdots$ & -0.29 & 0.13 & 2.82 & 8.11 & 2.01 \\
\hline & CDMSS & $\ldots$ & -0.26 & 1.08 & 5.84 & 20.99 & 4.19 \\
\hline & CDMP3 & $\cdots$ & 0.09 & 0.01 & 0.92 & 1.54 & 0.87 \\
\hline & CDMP3 & $\begin{array}{l}\cdots \\
\cdots\end{array}$ & 0.04 & 0.01 & 0.97 & 2.92 & 0.84 \\
\hline & CDMP3 & $\cdots$ & 0.09 & 0.07 & 1.89 & 6.60 & 1.69 \\
\hline \multirow{3}{*}{$\begin{array}{l}\text { soft } \ldots \ldots \ldots \ldots \ldots \ldots \\
\text { hard } \ldots \ldots \ldots \ldots \ldots \ldots\end{array}$} & XCDM & $\ldots$ & 0.08 & 0.02 & 1.13 & 2.91 & 1.01 \\
\hline & $\mathrm{XCDM}$ & $\cdots$ & 0.05 & 0.02 & 1.27 & 8.83 & 0.97 \\
\hline & $\mathrm{XCDM}$ & $\cdots$ & 0.14 & 0.11 & 2.4 & 18.92 & 1.97 \\
\hline
\end{tabular}

a $0.5-1.0 \mathrm{keV}$.

b $1.0-2.4 \mathrm{keV}$.

c $0.5-2.4 \mathrm{keV}$.

excluded. For instance, for simulations of a $R O S A T$ field with the self-similar model CDMSS, the granularity is negative at -0.260 when the threshold is 7.53 counts pixel $^{-1}$, corresponding to $5 \sigma$ above the assumed background. It remains negative for thresholds up to $\sim 10.0$, then turns positive, and reaches 0.88 for a threshold of 20 and 2.596 when no threshold is imposed. There really is clustering of photons present in the simulation; negative granularity is an artifact of the choice of threshold in the presence of the high spatial resolution of the ROSAT detector.

Simple generalizations of the common measure of granularity can be invented by replacing mean squares by cumulant generating functions. Specifically, letting $X_{i}$ be the count in the $i$ th pixel out of $k$ pixels, we suggest calculating as a function of a parameter $\alpha$ the quantity

$$
W_{\alpha}=\frac{\log \left[(1 / k) \sum \exp \left(-\alpha X_{i}\right)\right]}{\exp (-\alpha)-1}
$$

Purely Poisson data give a curve $W_{\alpha}$ whose expected value is independent of $\alpha$. The presence of weak extended sources like our clusters introduce a systematic downward slope into the graph of $W$ versus $\alpha$. For high values of $\alpha$, drastically reduced influence is automatically given to high counts. Thus with any measure based on $W_{\alpha}$ it is not necessary to introduce an arbitrary cutoff for excluding pixels with high counts due to strong detectable sources; the sensitivity of the measure of granularity to the choice of threshold is removed. $W_{\alpha}$ is easily computed from the histogram counts themselves. Furthermore, the function emphasizes the low-count end of the distribution of most interest for studying diffuse background contributions.

As a specific measure of granularity based on $W_{\alpha}$, we propose the "alpha granularity," minus the slope of the graph of $W_{\alpha}$ between values of $\alpha$ equal to one over the median count and two over the median count. For non-Poissonian observations, the choice of the range for calculating the slope does affect the value of the measure. If, however, the range is held fixed throughout a series of comparisons, the value should not be systematically altered (as is the variance-based measure of granularity) by increases or decreases in superposed Poissondistributed noise.

For the pixel configuration of the Einstein simulations, the null distribution in the absence of any component but the background noise for the $\alpha$-granularity is close to normal with a mean of zero and a standard deviation of $\sim 0.145$. The variance-based measure of granularity with a $5 \sigma$ cutoff is also a null distribution with negligible bias; its standard deviation is $\sim 0.047$. The critical point for rejecting the null hypothesis of no non-Poissonian component with the variance-based measure in a one-sided test would be $7 \%$, so that Hamilton \& Helfand's value of $16 \%$ is clearly significantly different from zero, even though they did not show it to be so. The critical point for the $\alpha$-granularity would be $\sim 0.28$. These points all depend on the size and number of pixels and the levels of noise assumed.

Values of $\alpha$-granularity, labeled $W_{\alpha}$, are shown next to the granularities based on variances in column (4) of Table 5. The remaining columns show the means and variances of pixel counts without exclusion and the trimmed mean, the mean of the pixels above threshold on which the granularities are based. The $W_{\alpha}$ values highlight the contrasts between models for the Einstein simulations which are hinted at by the conventional granularities.

For the ROSAT simulations, the $W_{\alpha}$ values indicate very little non-Poissonian variation for $5 \mathrm{hr}$ exposures. This impression is sustained by the histograms of ROSAT pixel counts in Figure 10, comparable to the right-hand panels of Figure 9. The histograms on the left pertain to the soft band and those on the right pertain to the hard band. Beyond the vastly larger total photon counts for the self-similar model, there is little structure to be seen. Longer exposures or grosser binning is likely to be necessary to tease out contrasts between the models on the basis of pixel count distributions alone.

\section{PREDICTIONS FOR ROSAT}

As we have emphasized, contribution of low-mass clusters or groups is potentially high in the ROSAT bands. This is quite important as the clusters appears to be a significant population in this band. From Figure 1, we see that such clusters should be at moderate redshift and should therefore be extended. Their detectability must therefore be addressed carefully. Images expected from deep ( $5 \mathrm{hr}$ ) observations with the ROSAT PSPC detector have been simulated. From comparison of the ROSAT PSPC in Figure 8 with the Einstein IPC images in the 


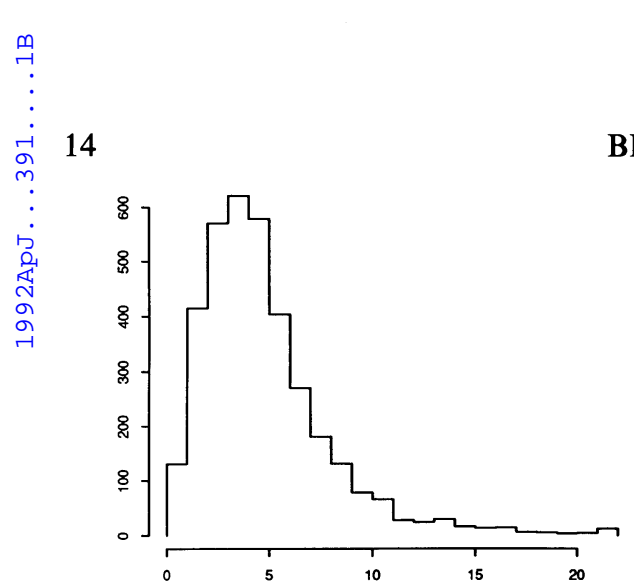

BLANCHARD, WACHTER, EVRARD, \& SILK

Vol. 391
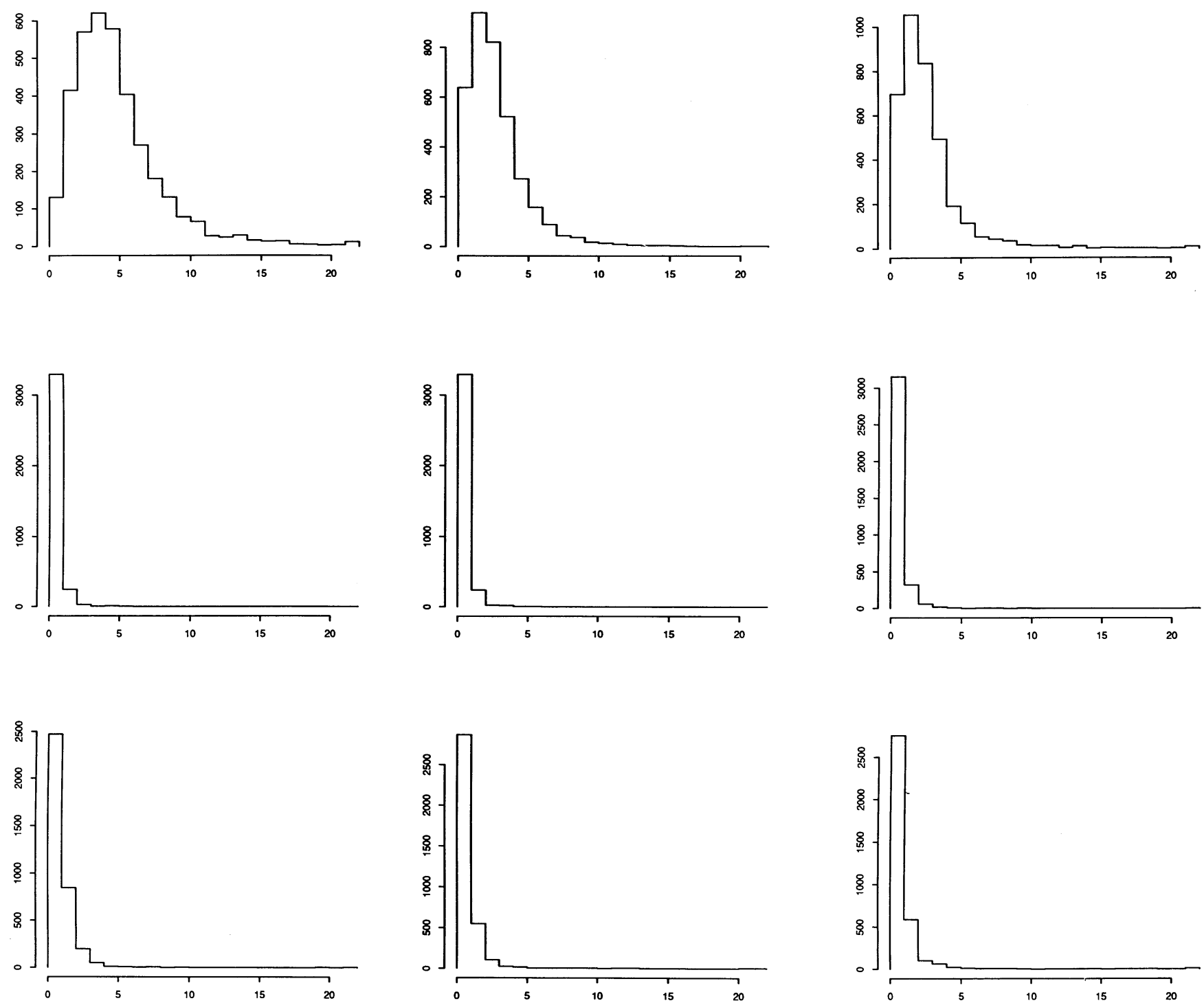

FIG. 10.-Histograms of simulated ROSAT pixel counts for sources only, soft band at left and hard band at right, CDMSS model at top, CDMP3 in middle, the XCDM at bottom.

figure, it is apparent that the sharper spatial resolution will make it easier to detect sources of cluster emission.

Figure 8 also shows the $R O S A T$ signal divided into three bands: $0.1-0.5,0.5-1.0$ and $1-2.4 \mathrm{keV}$. Results for all of the models are shown. Models CDMP3 and XCDM satisfy the constraints from the Einstein deep source counts. Histograms of counts are given in Figure 10 and the resultant granularity measures for these images are summarized in Table 5. The smaller pixel area, one-fourth the area of the ROSAT pixels, leads to smaller counts per pixel, and leaves the conventional variance-based granularities highly sensitive to the upper threshold, as we have mentioned. We might expect models CDMP3 and XCDM to differ somewhat in the ratio of soft to hard photons at the latter model probes somewhat lower masses, and hence lower temperatures, than the former (see Fig. 4). Unfortunately, Table 5 indicates that the ratio of soft to hard photons differs only by $\sim 5 \%$ between the models, being 0.95 for CDMP3 and 0.89 for XCDM. These ratios are heavily influenced by the background. Without the background, the ratios are 0.53 and 0.63 in the expected direction. The models cannot be distinguished by their granularity measures, either.

We have found that the self-similar model is inconsistent with the observations for the CDM model. As the CDM model is compatible with present observations of groups and clusters with respect to their optical properties (Moore, Frenk, \& White 1991), this seems to indicate the failure of the self-similar hypothesis. However, the temperature distribution function of clusters seems to be inconsistent with the CDM spectrum (Henry \& Arnaud 1991; Blanchard \& Silk 1991) so that only the XCDM seems consistent with present observations. On the other hand, care should be taken with regard to the actual detectability of the faint clusters, because of their intrinsic low temperature, faint surface brightness and large angular extension. ROSAT will greatly help clarify the situation. The present constraints are severe enough to predict that most of the contribution from clusters to the ROSAT band originates from objects that also contribute a similar amount in the $1-3 \mathrm{keV}$ Einstein band (see Fig. 4). In order to illustrate this point, we 
present the result expected in the ROSAT bands in the case of a model which is probably not compatible with present observational data: in the CDM model with $p=2$, the number of bright clusters $\left(L_{X} \geq 3 \times 10^{44}\right.$ ergs s $\left.^{-1}\right)$ agrees with the present observed number density, however, the number of clusters with luminosities $L_{X} \leq 10^{44}$ ergs $\mathrm{s}^{-1}$ is 3 times larger than the observed number, with a mean temperature of a few $\mathrm{keV}$. Actually these clusters correspond to very few detected sources in present X-ray surveys. Despite the fact that the number counts predict the presence of $\sim 10$ clusters per square degrees, only two are clearly visible on the corresponding map (not shown here), contributing $\sim 20 \%$ of the overall contribution from clusters in this band. These clusters are generally faint extended sources that appear clearly only when they are bright enough with a relatively moderate spatial extension. The "blotch" recently identified in a deep ROSAT NEP field (Hasinger et al. 1991) corresponds to this kind of object. The expected mean number of this kind of source is in the range 1-3 per square degree, but the number of fainter sources should be one order of magnitude larger. Actually most of the contribution of the sources results in a nearly uniform background. We emphasize that such clusters might contribute substantially to the background and have hitherto failed to have been detected.

\section{CONCLUSION}

Thermal emission from intergalactic gas in galaxy clusters is a well-understood process that is believed to contribute at least $10 \%-20 \%$ of the diffuse XRB at $2 \mathrm{keV}$. The fact that a significant fraction of all galaxies are in groups with velocity disper- sions corresponding to temperatures less than $2 \mathrm{keV}$ suggests that thermal emission may make a larger contribution at low $\mathrm{X}$-ray energies. Our approach in this paper has been to apply a viable formalism for the evolution of the mass function of galaxy groups and clusters to predict several of the observed quantities and to generate simulated deep images for both the Einstein IPC and the forthcoming ROSAT PSPC detectors. The fluctuation analysis of deep Einstein fields requires $50 \%$ of the 1-3 keV XRB to be produced by a source population in excess of 5000 objects per square degree. It has been argued that these objects are a population of active galactic nuclei or starburst galaxies. However, it is by no means obvious how many faint, extended galaxy groups or clusters could also be contributing.

That clusters contribute at redshift of $\sim 1$ or less is apparent from the fact that the richness of forming galaxy clusters increases with time in hierarchical models of large-scale structure formation. It follows that the larger abundance of galaxy groups at smaller masses and lower temperatures guarantees a substantial rise in flux below $2 \mathrm{keV}$ above that which might be naively inferred from the contribution of high-mass clusters to the $2-10 \mathrm{keV}$ band. ROSAT observations should shed new light on the contribution of bremsstrahlung from ionized gas in groups and clusters to the diffuse X-ray background.

This research has been supported in part at Berkeley by grants from NASA and NSF. A. E. gratefully acknowledges support by the Miller Foundation for Basic Research in Science at Berkeley.

\section{REFERENCES}

Barcons, X., \& Fabian, A. C. 1989, MNRAS, 237, 119

Blanchard, A., \& Silk, J. 1991, Proc. 26th Rencontres de Moriond in Astrophysics (Gif-sur-Yvette: Editions Frontières) (in press)

Bond, J. R., Cole, S., Efstathiou, G., \& Kaiser, N. 1991, ApJ, 370, 440

Boldt, E. 1987, Phys. Rep., 146, 215

Cole, S., \& Kaiser, N. 1989, MNRAS, 237, 1127

Collin-Souffrin, S. 1991, A\&A, 243, 5

Cowsik, R., \& Kobetich, E. J. 1972, ApJ, 177, 585

David, L., Arnaud, K. A., Forman, W., \& Jones, C. 1990, ApJ, 356, 32

Edge, A. C., Steward, G. C., Fabian, A. C., \& Arnaud, K. A. 1990, MNRAS, 245,559

Efstathiou, G., Frenk, C. S., White S. D. M., \& Davis, M. 1988, MNRAS, 235 , 715

Evrard, A. E. 1990, in Clusters of Galaxies, ed. W. R. Oegerle, M. J. Fitchett, \& L. Danly (Cambridge Univ. Press), 287

Evrard, A. E., \& Henry, J. P. 1991, ApJ, 383, 95

Fabian, A. C., George, I. M., Miyoshi, S., \& Rees, M. J. 1991, MNRAS, 242, 14P

Gioia, I. M., Maccacaro, T., Schild, R. E., Wolter, A., Stocke, J. T., Morris, S. L., \& Henry, J. P. 1990, ApJS, 72, 567

Giacconi, R., Gursky, H., Paolini, F., \& Rossi, B. 1962, Phys. Rev. Letters, 9, 439

Giacconi, R., et al. 1979, ApJ, 234, L1

Gunn, J. E., \& Gott, J. R. III. 1972, ApJ, 176, 1

Hamilton, T. T., \& Helfand, D. J. 1987, ApJ, 318, 93

Hamilton, T. T., Helfand, D. J., \& Wu, X. 1991, ApJ, 379, 576

Hasinger, G. 1992, Proc. Laredo Workshop, The X-ray Background, in press
Hasinger, G., Schmidt, M., \& Trümper, J. 1991, A\&A, 246, L1

Henry, J. P., \& Arnaud, K. 1992, ApJ, in press

Jones, W., \& Forman, C. 1984, ApJ, 276, 38

Kaiser, N. 1986, MNRAS, 222, 323

Marshall, F. E., Boldt, E. A., Holt, S. S., Miller, R. B., Mushotzky, R. F., Rose, L. A., Rothschild, R. E., \& Serlemitsos, P. J. 1980, ApJ, 235, 4

Mather, J. C., et al. 1990, ApJ, 354, L37

McCammon, D., \& Sanders, W. T. 1990, ARA\&A, 28, 657

McKee, J. D., Mushotzky, R. F., Boldt, E. A., Holt, S. S., Pravdo, S. H., \& Serlemitsos, P. J. 1980, ApJ, 242, 843

Morisawa, K., \& Takahara, F. 1989, PASJ, 41, 873

Moore, B., Frenk, C. S. F., \& White, S. D. M. 1991, preprint

Peebles, P. J. E. 1980, The Large-Scale Structure of the Universe (Princeton Univ. Press)

Penzias, A. A., \& Wilson, R. W. 1965, ApJ, 253, 1149

Piccinoti, G., Mushotzky, R. F., Boldt, E. A., Holt, S. S., Marshall, F. E. Serlemitsos, P. J., \& Shafer, R. A. 1982, ApJ, 253, 485

Press, W., \& Schechter, P. 1974, ApJ, 187, 425

Schaeffer, R., \& Silk, J. 1988, A\&A, 333, 509

Schwartz, D. A., \& Tucker, W. H. 1988, ApJ, 332, 157

Setti, G., \& Woltjer, L. 1991, A\&A, 224, L21

Silk, J., \& Tarter, J. 1973, Astrophys. J., 183, 387

Shanks, T., Georgantopoulos, I., Steward, G. C., Pounds, K. A., Boyle, B. J., \& Griffiths, R. E. 1991, Nature, 353, 315

Sołtan, 1991, MNRAS, 250, 241

Stottlemeyer, A. R., \& Boldt, E. A. 1984, ApJ, 279, 511

Wu, X., Hamilton, T. T., Helfand, D. J., \& Wang, Q. 1991, ApJ, 379, 564 